Jeffrey Streets · Gang Tian

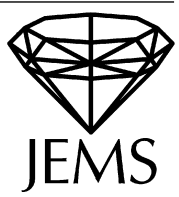

\title{
Hermitian curvature flow
}

Received February 13, 2009 and in revised form January 12, 2010

Abstract. We define a functional for Hermitian metrics using the curvature of the Chern connection. The Euler-Lagrange equation for this functional is an elliptic equation for Hermitian metrics. Solutions to this equation are related to Kähler-Einstein metrics, and are automatically KählerEinstein under certain conditions. Given this, a natural parabolic flow equation arises. We prove short time existence and regularity results for this flow, as well as stability for the flow near KählerEinstein metrics with negative or zero first Chern class.

\section{Introduction}

In this paper we introduce a new curvature evolution equation on compact complex manifolds. Specifically, given $\left(M^{2 n}, g, J\right)$ a manifold with integrable complex structure $J$ and Hermitian metric $g$, let $\nabla$ denote the Chern connection of $g$, which is a metric compatible connection with torsion $T$ [12]. Let $\Omega$ denote the curvature of $\nabla$. Define

$$
S_{i \bar{j}}=\left(\operatorname{tr}_{\omega} \Omega\right)_{i \bar{j}}=g^{k \bar{l}} \Omega_{k \bar{l} i \bar{j}}
$$

and let $s=g^{i} \bar{j} S_{i \bar{j}}$ be the scalar Chern curvature. Furthermore let $w_{i}=g^{j \bar{k}} T_{i j \bar{k}}$ denote the trace of the torsion. Consider the functional

$$
\mathbb{F}(g)=\frac{\int_{M}\left[s-\frac{1}{4}|T|^{2}-\frac{1}{2}|w|^{2}\right] d V}{\left(\int_{M} d V\right)^{(n-1) / n}} .
$$

As we will see in Section 3 this is the unique functional yielding $\stackrel{\circ}{S}$, the traceless part of $S$, as the traceless component of the second order terms in the associated Euler-Lagrange equation. Moreover, the form of the Euler-Lagrange equation suggests a flow equation in the same way that Ricci flow is suggested by the usual Hilbert functional. In particular we define an evolution equation

$$
\frac{\partial}{\partial t} g=-S+Q
$$

where $Q=Q(T)$ is a certain quadratic polynomial in the torsion $T$ of $\nabla$ which is made

J. Streets, G. Tian: Fine Hall, Princeton University, Princeton, NJ 08544, USA; e-mail: jstreets@math.princeton.edu, tian@math.princeton.edu 
precise in Section 3. We call equation (2) Hermitian curvature flow (HCF). Of course now it is known that Ricci flow is indeed the gradient flow of the lowest eigenvalue of a certain Schrödinger operator, although a corresponding statement for HCF is not yet known. It is also possible to write HCF in terms of Hodge-type operators. In particular, if $\omega(t)$ denotes the Kähler form of the time varying metric, then it satisfies the equation

$$
\left.\frac{\partial}{\partial t} \omega=-\left(\partial_{g}^{*} \partial \omega-\partial \partial_{g}^{*} \omega-\frac{\sqrt{-1}}{2} \partial \bar{\partial} \log \operatorname{det} g-2 \sqrt{-1}\left(\bar{\partial}_{g}^{*} \omega\right\lrcorner \bar{\partial} \omega\right)\right)+Q^{\prime}
$$

where $Q^{\prime}$ is a distinct fixed quadratic expression in the torsion.

We observe that when a solution $g(t)$ to HCF exists, the metric is Hermitian with respect to the fixed complex structure $J$ for all time. Secondly, we will show that when the initial metric $g(0)$ is Kähler, then the solution $g(t)$ is Kählerian and consequently the solution to HCF is given by Kähler-Ricci flow. Thirdly, we prove that certain static solutions are Kähler-Einstein metrics. It will be a very interesting problem to classify all static solutions. It is possible that most of them are Kähler. Hence, in some sense, this new flow evolves Hermitian metrics towards Kähler metrics.

Next we will prove a local existence theorem for HCF and develop some regularity properties for this flow. In particular we derive higher order derivative estimates in the presence of a curvature bound. These results are summarized in the following theorem.

Theorem 1.1. Let $\left(M^{2 n}, g_{0}, J\right)$ be a complex manifold with Hermitian metric $g_{0}$. There exists a constant $c(n)$ depending only on the dimension such that there exists a unique solution $g(t)$ to $\mathrm{HCF}$ for

$$
t \in\left[0, \frac{c(n)}{\max \left\{|\Omega|_{C^{0}\left(g_{0}\right)},|\nabla T|_{C^{0}\left(g_{0}\right)},|T|_{C^{0}\left(g_{0}\right)}^{2}\right\}}\right] .
$$

Moreover, there exist constants $C_{m}$ depending only on $m$ such that the estimates

$$
\left|\nabla^{m} \Omega\right|_{C^{0}\left(g_{t}\right)},\left|\nabla^{m+1} T\right|_{C^{0}\left(g_{t}\right)} \leq \frac{C_{m} \max \left\{|\Omega|_{C^{0}\left(g_{0}\right)},|\nabla T|_{C^{0}\left(g_{0}\right)},|T|_{C^{0}\left(g_{0}\right)}^{2}\right\}}{t^{m / 2}}
$$

hold for all $t$ in the above interval. Moreover, the solution exists on a maximal time interval $[0, \tau)$, and if $\tau<\infty$ then

$$
\limsup _{t \rightarrow \tau} \max \left\{|\Omega|_{C^{0}\left(g_{t}\right)},|\nabla T|_{C^{0}\left(g_{t}\right)},|T|_{C^{0}\left(g_{t}\right)}\right\}=\infty .
$$

Finally, if $g_{0}$ is Kähler, then $g(t)$ is a solution to Kähler-Ricciflow.

In some sense, the simplest possible behavior for this flow should occur near KählerEinstein metrics, where we expect the flow to be not too much different from KählerRicci flow. In this direction, we prove a stability result for HCF around Kähler-Einstein metrics with negative or zero first Chern class. Specifically, we show 
Theorem 1.2. Let $\left(M^{2 n}, g, J\right)$ be a complex manifold with Kähler-Einstein metric $g$ and $c_{1}(M)<0$ or $c_{1}(M)=0$. Then there exists $\epsilon=\epsilon(g)$ so that if $\widetilde{g}$ is a Hermitian metric on M compatible with $J$ and $|\widetilde{g}-g|_{C \infty}<\epsilon$ then the solution to $H C F$ with initial condition $\tilde{g}$ exists for all time and converges to a Kähler-Einstein metric.

It is important to note here that these theorems apply to a wide class of equations. In particular, consider

$$
\frac{\partial}{\partial t} g=-S+Q
$$

where $Q$ is any $(1,1)$-tensor which is a quadratic expression of the torsion. As mentioned above, in this paper we isolate a specific choice of $Q$ based on a certain unique functional introduced in Section 3, but Theorems 1.1 and 1.2 apply to solutions of 33, where $Q$ is any quadratic expression in the torsion. Depending on the ultimate goal one has, different choices of $Q$ may be appropriate.

There are two natural directions which motivate defining this flow. First, given all of the success of Ricci flow it is natural to study it on complex manifolds. However, it is usually the case that the Ricci tensor of a Hermitian metric is not $(1,1)$, and thus the Hermitian condition for the metric is not preserved. Thus the Ricci flow is not the best tool for studying complex geometry which is not already Kähler. The tensor $S$ is a natural $(1,1)$ curvature tensor associated to a Hermitian metric which differs from the Ricci tensor by torsion terms, meaning that it equals the Ricci tensor in the Kähler setting. Moreover, the operator $g \mapsto S(g)$ is strictly elliptic, giving HCF nice existence properties. Thus from this perspective HCF is the right analogue of Ricci flow for Hermitian geometry.

The second motivation, and actually our original motivation for HCF, is that it serves as a "holonomy flow". If one looks on the level of the Kähler form and asks for a parabolic flow which preserves the Hermitian condition and is stationary on Kähler manifolds, HCF comes up quite naturally. There is the side effect that one ends up looking not just for Kähler metrics, but Kähler-Einstein metrics. Given the excellent existence properties of the Kähler-Ricci flow, this is an acceptable price to pay. Indeed, other natural analytic approaches to this question which strictly look for Kähler metrics among Hermitian metrics (see for instance [19]) yield equations which are not elliptic. In [4] it is shown that if the usual Ricci-type curvature of the Chern connection is a nonzero scalar multiple of the metric, then the metric is automatically Kähler-Einstein. However, this Ricci tensor is not in general $(1,1)$, so from the perspective of Hermitian geometry, especially defining a flow of Hermitian metrics, this condition is not natural.

Here is an outline of the rest of the paper. In Section 2 we define all of the relevant objects and notation and provide various curvature formulas. In Section 3 we discuss the Hermitian Hilbert functional. Section 4 gives the definition of HCF and provides various equivalent formulations using Hodge-type operators and the Levi-Civita connection. In Sections 5-7 we prove existence and regularity properties for HCF. In Section 8 we prove the stability result for HCF around Kähler-Einstein metrics. We conclude in Section 9 with a discussion of some related questions. Section 10 is an appendix containing various useful calculations related to Hermitian geometry. 


\section{Differential operators on Hermitian manifolds}

Let $\left(M^{2 n}, g, J\right)$ be a complex manifold with a Hermitian metric $g$. In particular $J$ : $T M \rightarrow T M$ is an integrable almost complex structure, i.e.

$$
N_{J}(X, Y):=[J X, J Y]-J[J X, Y]-J[X, J Y]-[X, Y]=0
$$

for all $X, Y \in T M_{p}$. Furthermore

$$
g(u, v)=g(J u, J v) .
$$

This equation is written in complex coordinates as

$$
g_{i j}=g_{\overline{i j}}=0, \quad g_{i \bar{j}}=g_{\bar{j} i}=\bar{g}_{\bar{i} j} .
$$

Recall the Chern connection $\nabla$. In complex coordinates, the nonvanishing components of the connection are given by

$$
\Gamma_{i j}^{k}=g^{k \bar{l}} \partial_{i} g_{j \bar{l}} .
$$

This connection is compatible with $g$, but has torsion $T$. In particular in complex coordinates we have

$$
T_{i j}^{k}=g^{k \bar{l}}\left(\partial_{i} g_{j \bar{l}}-\partial_{j} g_{i \bar{l}}\right) .
$$

Also, there is a natural trace of the torsion

$$
w_{i}=T_{i j}^{j} .
$$

As we will see in the next section, $w$ is just a multiple of $\bar{\partial}^{*} \omega$ but this separate definition will be useful to us. We will also need certain quadratic expressions in the torsion. Specifically let

$$
\begin{aligned}
Q_{i \bar{j}}^{1} & =g^{k \bar{l}} g^{m \bar{n}} T_{i k \bar{n}} T_{\overline{j l} m}, \\
Q_{i \bar{j}}^{2} & =g^{k \bar{l}} g^{m \bar{n}} T_{\bar{l} \bar{n} i} T_{k m \bar{j}}, \\
Q_{i \bar{j}}^{3} & =g^{k \bar{l}} g^{m \bar{n}} T_{i k \bar{l}} T_{\bar{j} \bar{n} m}, \\
Q_{i \bar{j}}^{4} & =\frac{1}{2} g^{k \bar{k}} g^{m \bar{n}}\left(T_{m k \bar{l}} T_{\bar{n} \bar{j} i}+T_{\bar{n} \bar{l} k} T_{m i \bar{j}}\right) .
\end{aligned}
$$

Note that each $Q^{i}$ is a real symmetric $(1,1)$-tensor. The covariant derivatives of torsion also satisfy an identity.

Lemma 2.1. Given g a Hermitian metric,

$$
\nabla_{i} T_{j k \bar{l}}+\nabla_{k} T_{i j \bar{l}}+\nabla_{j} T_{k i \bar{l}}=T_{i j}^{p} T_{k p \bar{l}}+T_{j k}^{p} T_{i p \bar{l}}+T_{k i}^{p} T_{j p \bar{l}} .
$$


Proof. We directly compute

$$
\begin{aligned}
\nabla_{i} T_{j k \bar{l}}+\nabla_{k} T_{i \bar{l} \bar{l}}+\nabla_{j} T_{k i \bar{l}}= & \partial_{i} T_{j k \bar{l}}+\partial_{k} T_{i j \bar{l}}+\partial_{j} T_{k i \bar{l}}-\Gamma_{i j}^{p} T_{p k \bar{l}}-\Gamma_{i k}^{p} T_{j p \bar{l}}-\Gamma_{k i}^{p} T_{p j \bar{l}} \\
& -\Gamma_{k j}^{p} T_{i p \bar{l}}-\Gamma_{j k}^{p} T_{p i \bar{l}}-\Gamma_{j i}^{p} T_{k p \bar{l}} \\
= & T_{i j}^{p} T_{k p \bar{l}}+T_{j k}^{p} T_{i p \bar{l}}+T_{k i}^{p} T_{j p \bar{l}} .
\end{aligned}
$$

Next we collect some useful formulas for the Chern curvature. In particular, let $\Omega$ denote the curvature of the Chern connection and let $S$ be the trace, i.e.

$$
S_{\alpha \bar{\beta}}=\left(\operatorname{tr}_{\omega} \Omega\right)_{\alpha \bar{\beta}}=g^{\mu \bar{\nu}} \Omega_{\mu \bar{\nu} \alpha \bar{\beta}} .
$$

Further, let

$$
s=g^{\alpha \bar{\beta}} S_{\alpha \bar{\beta}} .
$$

We will let $P$ denote the trace of the transpose of $\Omega$, i.e.

$$
P_{\alpha \bar{\beta}}=g^{\mu \bar{v}} \Omega_{\alpha \bar{\beta} \mu \bar{v}} .
$$

In the Kähler case $S_{\alpha \bar{\beta}}=P_{\alpha \bar{\beta}}$ is the Ricci curvature and $s=r$ is the scalar curvature.

Lemma 2.2. Given g a Hermitian metric we have

$$
S_{j \bar{k}}=-g^{l \bar{m}} g_{j \bar{k}, l \bar{m}}+g^{l \bar{m}} g^{p \bar{q}} g_{p \bar{k}, \bar{m}} g_{j \bar{q}, l} .
$$

Proof. First of all we have

$$
\Omega_{l \bar{m} j \bar{k}}=-g_{\bar{k} p} \partial_{\bar{m}}\left(g^{p \bar{q}} \partial_{l} g_{j \bar{q}}\right)=-g_{j \bar{k}, l \bar{m}}+g^{p \bar{q}} g_{p \bar{k}, \bar{m}} g_{j \bar{q}, l} .
$$

Thus

$$
S_{j \bar{k}}=g^{l \bar{m}} \Omega_{l \bar{m} j \bar{k}}=-g^{l \bar{m}} g_{j \bar{k}, l \bar{m}}+g^{l \bar{m}} g^{p \bar{q}} g_{p \bar{k}, \bar{m}} g_{j \bar{q}, l}
$$

as required.

Lemma 2.3 (Bianchi Identity). For $X, Y, Z \in T_{x}(M)$ we have

$$
\begin{aligned}
& \Sigma\{\Omega(X, Y) Z\}=\Sigma\left\{T(T(X, Y), Z)+\nabla_{X} T(Y, Z)\right\} \\
& \Sigma\left\{\nabla_{X} \Omega(Y, Z)+\Omega(T(X, Y), Z)\right\}=0,
\end{aligned}
$$

where $\Sigma$ denotes the cyclic sum over the three vectors.

Lemma 2.4. Given g a Hermitian metric we have

$$
P_{i \bar{j}}-S_{i \bar{j}}=g^{k \bar{l}}\left(\nabla_{\bar{l}} T_{k i \bar{j}}+\nabla_{i} T_{\overline{l j} k}\right) .
$$

Proof. We compute using the Bianchi identity and the symmetries of the torsion:

$$
\Omega_{i \bar{j} k \bar{l}}=\Omega_{k \bar{j} i \bar{l}}+\nabla_{\bar{j}} T_{k i \bar{l}}=\Omega_{\bar{j} k \bar{l} i}+\nabla_{\bar{j}} T_{k i \bar{l}}=\Omega_{\bar{l} k \bar{j} i}+\nabla_{k} T_{\bar{l} j i}+\nabla_{\bar{j}} T_{k i \bar{l}} .
$$

Taking the trace and relabeling indices gives the result. 
Now we focus on Hodge operators associated to $g$. Let

$$
\omega(u, v)=-g(u, J v)
$$

be the Kähler form of $g$. In local complex coordinates we have

$$
\omega=\frac{\sqrt{-1}}{2} g_{i \bar{j}} d z^{i} \wedge d \bar{z}^{j}
$$

Let $\Lambda^{k}=\bigoplus_{p+q=k} \Lambda^{p, q}$ denote the usual decomposition of complex differential $k$-forms into forms of type $(p, q)$. The exterior differential $d$ decomposes into the operators $\partial$ and $\bar{\partial}$,

$$
\partial: \Lambda^{p, q} \rightarrow \Lambda^{p+1, q}, \quad \bar{\partial}: \Lambda^{p, q} \rightarrow \Lambda^{p, q+1} .
$$

Also the operator $d_{g}^{*}$, the $L^{2}$ adjoint of $d$, decomposes into $\partial_{g}^{*}$ and $\bar{\partial}_{g}^{*}$,

$$
\partial_{g}^{*}: \Lambda^{p+1, q} \rightarrow \Lambda^{p, q}, \quad \bar{\partial}_{g}^{*}: \Lambda^{p, q+1} \rightarrow \Lambda^{p, q} .
$$

Using these operators we can define the complex Laplacians

$$
\square_{\omega}=\partial_{g}^{*} \partial+\partial \partial_{g}^{*}: \Lambda^{p, q} \rightarrow \Lambda^{p, q}, \quad \bar{\square}_{\omega}=\bar{\partial}_{g}^{*} \bar{\partial}+\overline{\partial \partial}_{g}^{*}: \Lambda^{p, q} \rightarrow \Lambda^{p, q} .
$$

It is well known that the operator $\alpha \mapsto \square_{\omega} \alpha$ is a second order elliptic operator with symbol that of the Laplacian in complex coordinates [12]. Moreover, one has the formula

$$
\Delta_{d, g}=\square_{g}+\bar{\square}_{g}+\text { lower order terms. }
$$

However, we will be interested in the action of these operators on $\omega$ itself, so the terms which are lower order in (6) become highest order terms in this context. In the lemmas which follow we compute the action of these differential operators explicitly.

Lemma 2.5. Given g a Hermitian metric we have in complex coordinates

$$
\begin{aligned}
& \left(\partial_{g}^{*} \omega\right)_{\bar{k}}=\frac{\sqrt{-1}}{2} g^{p \bar{q}}\left(\partial_{\bar{q}} g_{p \bar{k}}-\partial_{\bar{k}} g_{p \bar{q}}\right), \\
& \left(\bar{\partial}_{g}^{*} \omega\right)_{j}=\frac{\sqrt{-1}}{2} g^{p \bar{q}}\left(\partial_{p} g_{j \bar{q}}-\partial_{j} g_{p \bar{q}}\right) .
\end{aligned}
$$

Proof. We compute using integration by parts. Given $\alpha \in \Lambda^{0,1}$ we have

$$
\begin{aligned}
\left(\partial_{g}^{*} \omega, \alpha\right) & =(\omega, \partial \alpha)=\int_{M} g^{\bar{k} l} g^{\bar{i} j}\left(\omega_{j \bar{k}} \overline{\partial \alpha_{i \bar{l}}}\right) d V \\
& =\frac{\sqrt{-1}}{2} \int_{M} g^{\bar{i} l}\left(\overline{\alpha_{\bar{l}, i}}\right) d V=-\frac{\sqrt{-1}}{2} \int_{M} \overline{\alpha_{\bar{l}}}\left[\partial_{\bar{i}}\left(g^{\bar{i} l} d V\right)\right] \\
& =-\frac{\sqrt{-1}}{2} \int_{M} \overline{\alpha_{\bar{l}}} d V\left[-g^{\bar{i} m} \partial_{\bar{i}} g_{m \bar{n}} g^{\bar{n} l}+g^{\bar{i} l} g^{p \bar{q}} \partial_{\bar{i}} g_{p \bar{q}}\right] .
\end{aligned}
$$

This gives the first formula, and the second follows analogously. 
Lemma 2.6. Given g a Hermitian metric we have in complex coordinates

$$
\left(\partial \partial_{g}^{*} \omega\right)_{j \bar{k}}=\frac{\sqrt{-1}}{2}\left[g^{p \bar{q}}\left(g_{p \bar{k}, \bar{q} j}-g_{p \bar{q}, \bar{k} j}\right)+g^{p \bar{q}} g^{r \bar{s}} g_{r \bar{q}, j}\left(g_{p \bar{s}, \bar{k}}-g_{p \bar{k}, \bar{s}}\right)\right] .
$$

Proof. In general for $\alpha \in \Lambda^{0,1}$ we have $(\partial \alpha)_{j \bar{k}}=\partial_{j} \alpha_{\bar{k}}$. Thus we compute using Lem$\mathrm{ma} 2.5$.

$$
\begin{aligned}
\left(\partial \partial_{g}^{*} \omega\right)_{j \bar{k}} & =\frac{\sqrt{-1}}{2} \partial_{j}\left(g^{p \bar{q}}\left(\partial_{\bar{q}} g_{p \bar{k}}-\partial_{\bar{k}} g_{p \bar{q}}\right)\right) \\
& =\frac{\sqrt{-1}}{2}\left[g^{p \bar{q}}\left(g_{p \bar{k}, \bar{q} j}-g_{p \bar{q}, \bar{k} j}\right)-g^{p \bar{m}} g_{\bar{m} n, j} g^{n \bar{q}}\left(g_{p \bar{k}, \bar{q}}-g_{p \bar{q}, \bar{k}}\right)\right] .
\end{aligned}
$$

The result follows.

Lemma 2.7. Given g a Hermitian metric we have in complex coordinates

$$
\begin{aligned}
\left(\partial_{g}^{*} \partial \omega\right)_{j \bar{k}}= & \frac{\sqrt{-1}}{2}\left[g^{p \bar{q}}\left(g_{p \bar{k}, j \bar{q}}-g_{j \bar{k}, p \bar{q}}\right)+g^{p \bar{q}} g^{r \bar{s}}\left(g_{p \bar{s}, \bar{q}}-g_{p \bar{q}, \bar{s}}\right)\left(g_{j \bar{k}, r}-g_{r \bar{k}, j}\right)\right. \\
& \left.+g^{p \bar{q}} g^{r \bar{s}} g_{j \bar{q}, \bar{s}}\left(g_{p \bar{k}, r}-g_{r \bar{k}, p}\right)+g^{p \bar{q}} g^{r \bar{s}} g_{p \bar{k}, \bar{s}}\left(g_{j \bar{q}, r}-g_{r \bar{q}, j}\right)\right] .
\end{aligned}
$$

Proof. First of all we know that

$$
(\partial \omega)_{i j \bar{k}}=\frac{\sqrt{-1}}{2}\left(g_{j \bar{k}, i}-g_{i \bar{k}, j}\right) .
$$

Now, we use the general formula for $\partial_{g}^{*}$ and compute

$$
\begin{aligned}
\left(\partial_{g}^{*} \partial \omega\right)_{j \bar{k}}= & -g_{j \bar{p}} g_{\bar{k} q}\left(\frac{\partial}{\partial \bar{z}^{m}}+\frac{1}{\bar{g}} \partial_{\bar{m}} \bar{g}\right)(\partial \omega)^{\overline{m p} q} \\
= & -\frac{\sqrt{-1}}{2}\left[g_{j \bar{p}} g_{\bar{k} q} \frac{\partial}{\partial \bar{z}^{m}}\left[g^{\bar{m} i} g^{\bar{p} r} g^{\bar{s} q}\left(g_{r \bar{s}, i}-g_{i \bar{s}, r}\right)\right]\right. \\
& \left.+g^{\bar{m} n} g^{p \bar{q}} g_{p \bar{q}, \bar{m}}\left(g_{j \bar{k}, n}-g_{n \bar{k}, j}\right)\right] \\
= & \frac{\sqrt{-1}}{2}\left[g^{p \bar{q}}\left(g_{p \bar{k}, j \bar{q}}-g_{j \bar{k}, p \bar{q}}\right)\right. \\
& +g_{j \bar{p}} g_{\bar{k} q}\left(g_{r \bar{s}, i}-g_{i \bar{s}, r}\right)\left[g^{\bar{m} u} g_{u \bar{v}, \bar{m}} g^{i \bar{v}} g^{\bar{p} r} g^{\bar{s} q}\right] \\
& +g_{j \bar{p}} g_{\bar{k} q}\left(g_{r \bar{s}, i}-g_{i \bar{s}, r}\right)\left[g^{\bar{m} i} g^{\bar{p} u} g_{u \bar{v}, \bar{m}} g^{\bar{v} r} g^{\bar{s} q}\right] \\
& +g_{j \bar{p}} g_{\bar{k} q}\left(g_{r \bar{s}, i}-g_{i \bar{s}, r}\right)\left[g^{\bar{m} i} g^{\bar{p} r} g^{\bar{s} u} g_{u \bar{v}, \bar{m}} g^{\bar{v} q}\right] \\
& \left.-g^{\bar{m} n} g^{p \bar{q}} g_{p \bar{q}, \bar{m}}\left(g_{j \bar{k}, n}-g_{n \bar{k}, j}\right)\right] \\
= & \frac{\sqrt{-1}}{2}\left[g^{p \bar{q}}\left(g_{p \bar{k}, j \bar{q}}-g_{j \bar{k}, p \bar{q}}\right)+g^{p \bar{q}} g^{r \bar{s}}\left(g_{p \bar{s}, \bar{q}}-g_{p \bar{q}, \bar{s}}\right)\left(g_{j \bar{k}, r}-g_{r \bar{k}, j}\right)\right. \\
& \left.+g^{p \bar{q}} g^{r \bar{s}} g_{j \bar{q}, \bar{s}}\left(g_{p \bar{k}, r}-g_{r \bar{k}, p}\right)+g^{p \bar{q}} g^{r \bar{s}} g_{p \bar{k}, \bar{s}}\left(g_{j \bar{q}, r}-g_{r \bar{q}, j}\right)\right] .
\end{aligned}
$$


Lemma 2.8. Given g a Hermitian metric we have in complex coordinates

$$
\left(\frac{\sqrt{-1}}{2} \partial \bar{\partial} \log \operatorname{det} g\right)_{j \bar{k}}=\frac{\sqrt{-1}}{2}\left(g^{p \bar{q}} \partial_{j} \partial_{\bar{k}} g_{p \bar{q}}-g^{p \bar{r}} \partial_{j} g_{\bar{r} s} g^{s \bar{q}} \partial_{\bar{k}} g_{p \bar{q}}\right) \text {. }
$$

Proof. We compute directly in coordinates:

$$
\begin{aligned}
\left(\frac{\sqrt{-1}}{2} \partial \bar{\partial} \log \operatorname{det} g\right)_{j \bar{k}} & =\frac{\sqrt{-1}}{2} \partial_{j}\left(g^{p \bar{q}} \partial_{\bar{k}} g_{p \bar{q}}\right) \\
& =\frac{\sqrt{-1}}{2}\left(g^{p \bar{q}} \partial_{j} \partial_{\bar{k}} g_{p \bar{q}}-g^{p \bar{r}} \partial_{j} g_{\bar{r} s} g^{s \bar{q}} \partial_{\bar{k}} g_{p \bar{q}}\right) .
\end{aligned}
$$

Also in this section we introduce canonical coordinates for $g$. We know that if $g$ is not Kähler then we cannot choose complex coordinates so that all the first derivatives of $g$ vanish. However, we can always ensure that a certain symmetric part of the first derivatives vanishes. This is made clear in the lemma below.

Lemma 2.9. Given a point $p \in M$, there exist coordinates around $p$ so that

$$
g_{i \bar{j}}=\delta_{i j} \quad \text { and } \quad \partial_{i} g_{j \bar{k}}+\partial_{j} g_{i \bar{k}}=0 .
$$

Proof. Let $\left\{z_{i}\right\}$ be arbitrary complex coordinate functions around $p$ so that $z^{i}(p)=0$ for all $i$. We briefly change our point of view and consider the Hermitian metric $h$ associated to $g$. The coordinate expression for $h$ takes the form

$$
h=h_{i j} d z^{i} d \bar{z}^{j}
$$

where $h_{i j}=\bar{h}_{j i}$. Without loss of generality by a rotation and rescaling we can assume

$$
h_{i j}(p)=\delta_{i j}
$$

Define new coordinates $\left\{w^{i}\right\}$ by the equation

$$
w^{i}=z^{i}+\frac{1}{4} \sum_{j, k}\left(\frac{\partial}{\partial z^{k}} h_{i j}(p)+\frac{\partial}{\partial z^{j}} h_{i k}(p)\right) z^{j} z^{k}
$$

so that

$$
d w^{i}=d z^{i}+\frac{1}{2} \sum_{j, k}\left(\frac{\partial}{\partial z^{k}} h_{i j}(p)+\frac{\partial}{\partial z^{j}} h_{i k}(p)\right) z^{j} d z^{k} .
$$

Note also that (10) still holds in these coordinates. In these new coordinates write

$$
h=\widetilde{h}_{i j} d w^{i} d \bar{w}^{j} .
$$

It is clear that

$$
\tilde{h}_{i j}=h_{i j}-\frac{1}{2} \sum_{j, k}\left(\frac{\partial}{\partial z^{k}} h_{i j}(p)+\frac{\partial}{\partial z^{j}} h_{i k}(p)\right) z^{k}+\mathcal{O}\left(z^{2}\right) .
$$

The claim follows directly by differentiating. 


\section{The Hermitian Hilbert functional}

Let $\left(M^{2 n}, g, J\right)$ be a complex manifold. Consider the functional

$$
\mathbb{F}(g)=\frac{\int_{M}\left[s-\frac{1}{4}|T|^{2}-\frac{1}{2}|w|^{2}\right] d V}{\left(\int_{M} d V\right)^{(n-1) / n}} .
$$

Lemma 3.1. Let $g(a)$ be a one-parameter family of Hermitian metrics with variation $h$. Then

$$
\begin{aligned}
\frac{\partial}{\partial a} \mathbb{F}(g)=\left(\int_{M} d V\right)^{(1-n) / n} \int_{M}\left\langle h,-S+\frac{1}{2} Q^{1}-\frac{1}{4} Q^{2}-\frac{1}{2} Q^{3}+Q^{4}\right. \\
\left.+\left(s-\frac{1}{4}|T|^{2}-\frac{1}{2}|w|^{2}-\frac{n-1}{n} \frac{\left(\int_{M} s-\frac{1}{4}|T|^{2}-\frac{1}{2}|w|^{2}\right) d V}{\int_{M} d V}\right) g\right\rangle d V .
\end{aligned}
$$

Moreover, $\mathbb{F}$ is the unique second order functional which yields $\stackrel{\circ}{S}$ as the leading order term in the traceless part of the variational equation through Hermitian metrics.

Proof. Combining Lemmas $10.7+10.9$ we see

$$
\begin{aligned}
& \frac{\partial}{\partial a} \int_{M}\left[s-\frac{1}{4}|T|^{2}-\frac{1}{2}|w|^{2}\right] d V \\
& \quad=\int_{M}\left[\left\langle h,-S+\frac{1}{2} Q^{1}-\frac{1}{4} Q^{2}-\frac{1}{2} Q^{3}+Q^{4}\right\rangle+(\operatorname{tr} h)\left(s-\frac{1}{4}|T|^{2}-\frac{1}{2}|w|^{2}\right)\right] d V .
\end{aligned}
$$

Likewise we compute

$$
\frac{\partial}{\partial a}\left(\int_{M} d V\right)^{(n-1) / n}=\frac{n-1}{n} \int_{M} \operatorname{tr} h d V\left(\int_{M} d V\right)^{-1 / n} .
$$

Combining these two calculations gives the result. The uniqueness claim is also clear by inspection of the variational formulas in Lemmas $10.7+10.9$

Let

$$
Q=\frac{1}{2} Q^{1}-\frac{1}{4} Q^{2}-\frac{1}{2} Q^{3}+Q^{4}
$$

and let $K:=S-Q$. Note that

$$
k:=\operatorname{tr}_{g} K=s-\frac{1}{4}|T|^{2}-\frac{1}{2}|w|^{2} .
$$

We can rephrase the above situation in a very simple manner. In particular

$$
\mathbb{F}(g)=\int_{M} k d V
$$


and

$$
\frac{\partial}{\partial a} \mathbb{F}(g(a))=\int_{M}\left\langle h,-K+k g-\frac{n-1}{n} \frac{\int_{M} k d V}{\int_{M} d V} g\right\rangle d V,
$$

which is exactly analogous to the form of the gradient of the normalized Hilbert functional.

Definition 3.2. Given $\left(M^{2 n}, g, J\right)$ a complex manifold we say that $g$ is static if $g$ is critical for $\mathbb{F}$.

Proposition 3.3. Let $\left(M^{2 n}, g, J\right)$ be a complex manifold with $g$ static. Then

$$
K-\frac{1}{n} k g=0 .
$$

Also $k$ is a constant function. Finally, if $\mathbb{F}(g) \geq 0$ and $\int_{M} s d V_{g} \leq 0$ then $g$ is KählerEinstein.

Proof. The first property follows immediately by letting $h=K-(1 / n) k g$ in $(12)$. Next let $h=-\left(\Delta_{D} k\right) g$ where $\Delta_{D}$ means the Laplacian with respect to the Levi-Civita connection. Plugging this into 12 yields

$$
0=-\int_{M}\left(\Delta_{D} k\right) k d V=\int_{M}|d k|^{2} .
$$

To see the last claim we simply note that together the hypotheses imply

$$
0 \leq \mathbb{F}(g)=V^{-1 / n} k=V^{-1 / n}\left(s-\frac{1}{4}|T|^{2}-\frac{1}{2}|w|^{2}\right) \leq-\frac{1}{V^{1 / n}}|T|^{2},
$$

which implies $T \equiv 0$. If the torsion of $g$ vanishes, then $g$ is Kähler and moreover $K$ is given by the Ricci tensor of $g$, so $g$ is Kähler-Einstein.

To emphasize, $S$ is in a sense the only natural curvature tensor associated to a Hermitian metric which is a symmetric $(1,1)$-tensor and which is a second order elliptic operator. In seeking a functional which yields $S$ as the leading term in the Euler-Lagrange equation, $\mathbb{F}$ above is the only choice. We note that the functional $\int_{M} s d V_{g}$ was considered in [9]. Indeed, for this functional one finds automatically that critical points are Kähler-Einstein if the value of the functional is nonzero. However, there the leading term in the EulerLagrange equation is $P$, which is not an elliptic operator on Hermitian metrics. Finally, we remark that $\mathbb{F}$ is not the Hilbert functional restricted to Hermitian metrics. Indeed, a straightforward calculation (see also [8, line (33)]) shows that if $r$ denotes the usual scalar curvature,

$$
\int_{M} r d V_{g}=\int_{M} s-\frac{1}{4}|T|^{2}
$$

Therefore we see that $\mathbb{F}=\int_{M}\left(r-\frac{1}{2}|w|^{2}\right) d V_{g}$ restricted to Hermitian metrics. This bears a certain formal similarity to functionals related to renormalization group flows arising in physical models [13], [16], [17]. 


\section{Hermitian curvature flow}

In this section we give the definition of Hermitian curvature flow in terms of the Chern curvature. We then provide an equivalent definition using Hodge operators. In all the calculations below, $Q$ is defined by $(11)$.

Proposition 4.1. Let $\left(M^{2 n}, g, J\right)$ be a Hermitian manifold and let

$$
\Phi(g):=(S-Q)(g)
$$

Then $\Phi$ is a map

$$
\Phi: \Re \operatorname{Sym}^{1,1} T^{*} M \rightarrow \Re \operatorname{Sym}^{1,1} T^{*} M
$$

where $\mathfrak{R} \mathrm{Sym}^{1,1} T^{*} M$ are the real symmetric $(1,1)$-tensors. Moreover, $\Phi$ is a nonlinear second order elliptic operator.

Proof. It follows from Lemma 2.2 that $\Phi(g)_{i \bar{j}}=-g^{k \bar{l}} g_{i \bar{j}, k \bar{l}}+\mathcal{O}(\partial g)$ and so $\Phi$ is a nonlinear second order elliptic operator since $g$ is positive definite. Also, by definition each of the tensors $Q^{i}$ is a real symmetric $(1,1)$-tensor and thus $Q$ is. Lemma 2.2 implies that $S$ is also a real symmetric $(1,1)$-tensor. Therefore $\Phi(g)$ is a real symmetric $(1,1)$ tensor. The result follows.

Definition 4.2. Given $\left(M^{2 n}, J, g_{0}\right)$ a complex manifold with Hermitian metric $g_{0}$. We say that a one-parameter family of Hermitian metrics $g(t)$ is a solution to Hermitian curvature flow $(\mathrm{HCF})$ with initial condition $g_{0}$ if

$$
\begin{aligned}
\frac{\partial}{\partial t} g(t) & =-S(g(t))+Q(g(t)), \\
g(0) & =g_{0} .
\end{aligned}
$$

Next we compute a formula for HCF using Hodge operators. Define

$$
\Xi=S(\cdot, J \cdot)
$$

We will write $\Xi(g)$ using Hodge differentials. Let

$$
\left.\Psi(\omega):=\partial_{g}^{*} \partial \omega-\partial \partial_{g}^{*} \omega-\frac{\sqrt{-1}}{2} \partial \bar{\partial} \log \operatorname{det} g-2 \sqrt{-1}\left(\bar{\partial}_{g}^{*} \omega\right\lrcorner \bar{\partial} \omega\right) .
$$

We choose to isolate this term because, as we will see in the calculations below, it is a real $(1,1)$-form.

Proposition 4.3. Given g a Hermitian metric, we have

$$
\Xi(g)=\Psi(\omega)-\frac{\sqrt{-1}}{2}\left(2 Q_{j \bar{k}}^{4}+\frac{1}{2} Q_{j \bar{k}}^{2}\right) .
$$


Proof. Choose coordinates according to Lemma 2.9 so that at a fixed point $p \in M$,

$$
\partial_{i} g_{j \bar{k}}=-\partial_{j} g_{i \bar{k}}
$$

Using this we find that at the point $p$,

$$
\frac{1}{2} T_{i j \bar{k}}=\frac{1}{2}\left(\partial_{i} g_{j \bar{k}}-\partial_{j} g_{i \bar{k}}\right)=\partial_{i} g_{j \bar{k}}
$$

Next we compute a formula for $\Psi$ in coordinates. In particular we compute a formula for $\partial \bar{\partial} \log \operatorname{det} g$ :

$$
\begin{aligned}
\frac{\sqrt{-1}}{2}(\partial \bar{\partial} \log \operatorname{det} g)_{j \bar{k}} & =\frac{\sqrt{-1}}{2} \partial_{j}\left(g^{p \bar{q}} \partial_{\bar{k}} g_{p \bar{q}}\right)=\frac{\sqrt{-1}}{2}\left(g^{p \bar{q}} \partial_{j} \partial_{\bar{k}} g_{p \bar{q}}-g^{p \bar{r}} \partial_{j} g_{\bar{r} s} g^{s \bar{q}} \partial_{\bar{k}} g_{p \bar{q}}\right) \\
& =\frac{\sqrt{-1}}{2}\left(g^{p \bar{q}} g_{p \bar{q}, j \bar{k}}-\frac{1}{4} g^{p \bar{q}} g^{r \bar{s}} T_{j r \bar{q}} T_{\bar{k} \bar{s} p}\right) .
\end{aligned}
$$

Next we compute using Lemma 2.5

$$
\left.-2 \sqrt{-1}\left(\bar{\partial}_{g}^{*} \omega\right\lrcorner \bar{\partial} \omega\right)=-2 \sqrt{-1} g{ }^{p \bar{q}}\left(\bar{\partial}_{g}^{*} \omega\right)_{p}(\bar{\partial} \omega)_{\bar{q} j \bar{k}}=\frac{\sqrt{-1}}{2} g^{p \bar{q}} g^{r \bar{s}} T_{r p \bar{s}} T_{\bar{q} \bar{k} j}
$$

We now combine these calculations with Lemmas 2.6 and 2.7 to get

$$
\begin{aligned}
& \Psi(\omega)_{j \bar{k}}= \frac{\sqrt{-1}}{2}\left[-g^{p \bar{q}} g_{j \bar{k}, p \bar{q}}+\frac{1}{2} g^{p \bar{q}} g^{r \bar{s}}\left[2 T_{\bar{q} s} T_{r j \bar{k}}+2 T_{p r \bar{q}} T_{\bar{s} \bar{k} j}\right.\right. \\
&\left.\left.+T_{\overline{s q} j} T_{r p \bar{k}}+T_{\bar{s} \bar{k} p} T_{r j \bar{q}}-T_{j r \bar{q}} T_{\bar{k} \bar{s} p}+\frac{1}{2} T_{j r \bar{q}} T_{\bar{k} \bar{s} p}\right]\right] \\
&=\frac{\sqrt{-1}}{2}\left[-g^{p \bar{q}} g_{j \bar{k}, p \bar{q}}+\frac{1}{2} g^{p \bar{q}} g^{r \bar{s}}\left[2 T_{\overline{q s} p} T_{r j \bar{k}}+2 T_{p r \bar{q}} T_{\bar{s} \bar{k} j}+T_{\overline{s q} j} T_{r p \bar{k}}+\frac{1}{2} T_{j r \bar{q}} T_{\bar{k} \bar{s} p}\right]\right] .
\end{aligned}
$$

Likewise we have, from Lemma 2.2,

$$
\Xi(g)_{j \bar{k}}=\frac{\sqrt{-1}}{2}\left[-g^{p \bar{q}} g_{j \bar{k}, p \bar{q}}+\frac{1}{4} g^{p \bar{q}} g^{r \bar{s}} T_{j p \bar{s}} T_{\bar{k} \bar{q} r}\right] .
$$

The result follows by combining these calculations.

Corollary 4.4. The HCF equation is equivalent to

$$
\frac{\partial}{\partial t} \omega=-\Psi(\omega)+\frac{\sqrt{-1}}{2}\left(\frac{1}{2} Q^{1}+\frac{1}{4} Q^{2}-\frac{1}{2} Q^{3}+3 Q^{4}\right) .
$$

Proof. This follows immediately from the definition of HCF and Proposition 4.3 . 


\section{Short-time existence}

Proposition 5.1. Given $\left(M^{2 n}, J, g_{0}\right)$ a compact complex manifold, there exists a unique solution to HCF with initial condition $g_{0}$ on $[0, \epsilon)$ for some $\epsilon>0$.

Proof. Since the operator $\Phi(g)$ is strictly elliptic by Proposition 4.1, the HCF equation is strictly parabolic, and thus short-time existence and uniqueness follow from standard theory.

Proposition 5.2. Given $\left(M^{2 n}, J, g_{0}\right)$ a compact complex manifold with Kähler metric $g_{0}$, let $g(s)$ denote the solution to HCF with initial condition $g_{0}$, which exists on $[0, T)$. Then for all $t \in[0, T), g(t)$ is Kähler, and is a solution to Kähler-Ricciflow.

Proof. Let $\widetilde{g}(t)$ be the solution to Kähler-Ricci flow with initial condition $g_{0}$. Ricci flow preserves the Kähler condition, thus $\widetilde{g}(t)$ is Kähler for all time, hence $\widetilde{T}=d \widetilde{\omega}=0$ and $\widetilde{Q}=0$. It follows that $\widetilde{\mathrm{Rc}}=\widetilde{S}=\widetilde{S}-\widetilde{Q}$ and so

$$
\frac{\partial}{\partial t} \widetilde{g}(t)=-\widetilde{\mathrm{Rc}}=-\widetilde{S}+\widetilde{Q}
$$

Thus $\widetilde{g}(t)$ is a solution to HCF with initial condition $g_{0}$. Since solutions to HCF are unique, it follows that $\widetilde{g}(t)=g(t)$ for all time and hence $g(t)$ is Kähler for all time and solves Kähler-Ricci flow.

\section{Evolution equations}

Lemma 6.1. For a solution to HCF we have

$$
\begin{aligned}
\frac{\partial}{\partial t} \Omega_{i \bar{j} k \bar{l}}= & \Delta \Omega_{i \bar{j} k \bar{l}}+g^{m \bar{n}}\left(T_{\bar{n} \bar{j}}^{\bar{p}} \nabla_{m} \Omega_{i \bar{p} k \bar{l}}+T_{m i}^{p} \nabla_{\bar{j}} \Omega_{p \bar{n} k \bar{l}}\right) \\
& +g^{m \bar{n}}\left(\Omega_{i \bar{j} m}^{p} \Omega_{p \bar{n} k \bar{l}}+\Omega_{m \bar{n} \bar{j}}^{\bar{p}} \Omega_{i \bar{p} k \bar{l}}+\Omega_{m \bar{j} k}^{p} \Omega_{i \bar{n} p \bar{l}}+\Omega_{m \overline{j l}}^{\bar{p}} \Omega_{i \bar{n} k \bar{p}}\right) \\
& -\Omega_{i \bar{j} k}^{m}\left(S_{m \bar{l}}-Q_{m \bar{l}}\right)-\nabla_{\bar{j}} \nabla_{i} Q_{k \bar{l}} .
\end{aligned}
$$

Proof. First consider the term $Q$ in the evolution of $g$. Using Lemma 10.2 we see that this contributes

$$
\Omega_{i \bar{j} k}^{m} Q_{m \bar{l}}-\nabla_{\bar{j}} \nabla_{i} Q_{k \bar{l}}
$$

to the evolution of $\Omega_{i \bar{j} k \bar{l}}$. Next we consider the contribution of the term $-S$ in the evolution of $g$. Using Lemma 10.2 we see that the evolution $\frac{\partial}{\partial t} g=-S$ yields

$$
\frac{\partial}{\partial t} \Omega_{i \bar{j} k \bar{l}}=-\Omega_{i \bar{j} k}^{m} S_{m \bar{l}}+\nabla_{\bar{j}} \nabla_{i} S_{k \bar{l}} .
$$

Now we must apply the second Bianchi identity. We have

$$
\begin{aligned}
\nabla_{\bar{j}}\left(\nabla_{i} S_{k \bar{l}}\right) & =\nabla_{\bar{j}} g^{m \bar{n}}\left(\nabla_{i} \Omega_{m \bar{n} k \bar{l}}\right)=\nabla_{\bar{j}} g^{m \bar{n}}\left(\nabla_{m} \Omega_{i \bar{n} k \bar{l}}+T_{m i}^{p} \Omega_{p \bar{n} k \bar{l}}\right) \\
& =g^{m \bar{n}}\left(\nabla_{\bar{j}} \nabla_{m} \Omega_{i \bar{n} k \bar{l}}+\nabla_{\bar{j}} T_{m i}^{p} \Omega_{p \bar{n} k \bar{l}}+T_{m i}^{p} \nabla_{\bar{j}} \Omega_{p \bar{n} k \bar{l}}\right) .
\end{aligned}
$$


Next we commute covariant derivatives to get

$$
\begin{aligned}
g^{m \bar{n}} \nabla_{\bar{j}} \nabla_{m} \Omega_{i \bar{n} k \bar{l}}= & g^{m \bar{n}}\left(\nabla_{m} \nabla_{\bar{j}} \Omega_{i \bar{n} k \bar{l}}+\Omega_{m \bar{j} i}^{p} \Omega_{p \bar{n} k \bar{l}}+\Omega_{m \bar{j} \bar{n}}^{\bar{p}} \Omega_{i \bar{p} k \bar{l}}\right. \\
& \left.+\Omega_{m \bar{j} k}^{p} \Omega_{i \bar{n} p \bar{l}}+\Omega_{m \overline{j l}}^{\bar{p}} \Omega_{i \bar{n} k \bar{p}}\right) .
\end{aligned}
$$

Finally, we apply the Bianchi identity again to get

$$
\begin{aligned}
g^{m \bar{n}} \nabla_{m} \nabla_{\bar{j}} \Omega_{i \bar{n} k \bar{l}} & =g^{m \bar{n}} \nabla_{m}\left(\nabla_{\bar{n}} \Omega_{i \bar{j} k \bar{l}}+T_{\bar{n} \bar{j}}^{\bar{p}} \Omega_{i \bar{p} k \bar{l}}\right) \\
& =\Delta \Omega_{i \bar{j} k \bar{l}}+g^{m \bar{n}}\left(\nabla_{m} T_{\bar{n} \bar{j}}^{\bar{p}} \Omega_{i \bar{p} k \bar{l}}+T_{\bar{n} \bar{j}}^{\bar{p}} \nabla_{m} \Omega_{i \bar{p} k \bar{l}}\right) .
\end{aligned}
$$

Combining these calculations yields

$$
\begin{aligned}
\frac{\partial}{\partial t} \Omega_{i \bar{j} k \bar{l}}= & \Delta \Omega_{i \bar{j} k \bar{l}}+g^{m \bar{n}}\left(\nabla_{m} T_{\bar{n} \bar{j}}^{\bar{p}} \Omega_{i \bar{p} k \bar{l}}+T_{\bar{n} \bar{j}}^{\bar{p}} \nabla_{m} \Omega_{i \bar{p} k \bar{l}}+\nabla_{\bar{j}} T_{m i}^{p} \Omega_{p \bar{n} k \bar{l}}+T_{m i}^{p} \nabla_{\bar{j}} \Omega_{p \bar{n} k \bar{l}}\right) \\
& +g^{m \bar{n}}\left(\Omega_{m \bar{j} i}^{p} \Omega_{p \bar{n} k \bar{l}}+\Omega_{m \bar{j} \bar{n}}^{\bar{p}} \Omega_{i \bar{p} k \bar{l}}+\Omega_{m \bar{j} k}^{p} \Omega_{i \bar{n} p \bar{l}}+\Omega_{m \overline{j l}}^{\bar{p}} \Omega_{i \bar{n} k \bar{p}}\right)-\Omega_{i \bar{j} k}^{m} S_{m \bar{l}} .
\end{aligned}
$$

Now we can apply the Bianchi identity to the terms

$$
\Omega_{m \bar{j} \bar{n}}^{\bar{p}}=\Omega_{m \bar{n} \bar{j}}^{\bar{p}}+\nabla_{m} T_{\bar{j} \bar{n}}^{\bar{p}} \quad \text { and } \quad \Omega_{m \bar{j} i}^{p}=\Omega_{i \bar{j} m}^{p}+\nabla_{\bar{j}} T_{i m}^{p} .
$$

Plugging these two in yields

$$
\begin{aligned}
\frac{\partial}{\partial t} \Omega_{i \bar{j} k \bar{l}}= & \Delta \Omega_{i \bar{j} k \bar{l}}+g^{m \bar{n}}\left(T_{\bar{n} \bar{j}}^{\bar{p}} \nabla_{m} \Omega_{i \bar{p} k \bar{l}}+T_{m i}^{p} \nabla_{\bar{j}} \Omega_{p \bar{p} k \bar{l}}\right) \\
& +g^{m \bar{n}}\left(\Omega_{i \bar{j} m}^{p} \Omega_{p \bar{n} k \bar{l}}+\Omega_{m \bar{n} \bar{j}}^{\bar{p}} \Omega_{i \bar{p} k \bar{l}}+\Omega_{m \bar{j} k}^{p} \Omega_{i \bar{n} p \bar{l}}+\Omega_{m \overline{j l}}^{\bar{p}} \Omega_{i \bar{n} k \bar{p}}\right)-\Omega_{i \bar{j} k}^{m} S_{m \bar{l}} .
\end{aligned}
$$

Combining this with the above terms gives the result.

Lemma 6.2. For a solution to HCF we have

$$
\begin{aligned}
\frac{\partial}{\partial t} T_{i j \bar{k}}= & \Delta T_{i j \bar{k}}+g^{m \bar{n}}\left[T_{j i}^{p} \nabla_{\bar{n}} T_{m p \bar{k}}+\nabla_{\bar{n}} T_{m j}^{p} T_{i p \bar{k}}+T_{m j}^{p} \nabla_{\bar{n}} T_{i p \bar{k}}\right. \\
& \left.+\nabla_{\bar{n}} T_{i m}^{p} T_{j p \bar{k}}+T_{i m}^{p} \nabla_{\bar{n}} T_{j p \bar{k}}\right] \\
& +g^{m \bar{n}}\left[\Omega_{\bar{n} j m}^{p} T_{i p \bar{k}}+\Omega_{\bar{n} j \bar{k}}^{\bar{p}} T_{i m \bar{p}}-\Omega_{\bar{n} i m}^{p} T_{j p \bar{k}}-\Omega_{\bar{n} i \bar{k}}^{\bar{p}} T_{j m \bar{p}}\right. \\
& \left.-\Omega_{p \bar{n} m \bar{k}} T_{j i}^{p}\right]-T_{i j}^{p}\left(S_{p \bar{k}}-Q_{p \bar{k}}\right)+\nabla_{i} Q_{j \bar{k}}-\nabla_{j} Q_{i \bar{k}} .
\end{aligned}
$$

Proof. First we compute the contribution from the term $Q$ in the evolution of $g$. In particular using Lemma 10.4 this yields

$$
\nabla_{i} Q_{j \bar{k}}-\nabla_{j} Q_{i \bar{k}}+T_{i j}^{p} Q_{p \bar{k}} .
$$

Next we focus on the term $-S$. Applying Lemma 10.4 we obtain

$$
\nabla_{j} S_{i \bar{k}}-\nabla_{i} S_{j \bar{k}}-T_{i j}^{p} S_{p \bar{k}}
$$


Now using the Bianchi identity we rewrite

$$
\begin{aligned}
& \nabla_{j} S_{i \bar{k}}=g^{m \bar{n}} \nabla_{j} \Omega_{m \bar{n} i \bar{k}}=g^{m \bar{n}} \nabla_{j}\left(\Omega_{i \bar{n} m \bar{k}}+\nabla_{\bar{n}} T_{i m \bar{k}}\right), \\
& \nabla_{i} S_{j \bar{k}}=g^{m \bar{n}} \nabla_{i}\left(\Omega_{j \bar{n} m \bar{k}}+\nabla_{\bar{n}} T_{j m \bar{k}}\right) .
\end{aligned}
$$

Combining these yields

$$
\frac{\partial}{\partial t} T_{i j \bar{k}}=g^{m \bar{n}}\left(\nabla_{j} \Omega_{i \bar{n} m \bar{k}}-\nabla_{i} \Omega_{j \bar{n} m \bar{k}}+\nabla_{j} \nabla_{\bar{n}} T_{i m \bar{k}}-\nabla_{i} \nabla_{\bar{n}} T_{j m \bar{k}}\right)-T_{i j}^{p} S_{p \bar{k}} .
$$

Applying the Bianchi identity again yields

$$
g^{m \bar{n}}\left(\nabla_{j} \Omega_{i \bar{n} m \bar{k}}-\nabla_{i} \Omega_{j \bar{n} m \bar{k}}\right)=-g^{m \bar{n}} T_{j i}^{p} \Omega_{p \bar{n} m \bar{k}} .
$$

Also, we commute derivatives:

$$
\begin{aligned}
& \nabla_{j} \nabla_{\bar{n}} T_{i m \bar{k}}=\nabla_{\bar{n}} \nabla_{j} T_{i m \bar{k}}+\Omega_{\bar{n}{ }_{j i}}^{p} T_{p m \bar{k}}+\Omega_{\bar{n} j m}^{p} T_{i p \bar{k}}+\Omega_{\bar{n} j \bar{k}}^{\bar{p}} T_{i m \bar{p}}, \\
& \nabla_{i} \nabla_{\bar{n}} T_{j m \bar{k}}=\nabla_{\bar{n}} \nabla_{i} T_{j m \bar{k}}+\Omega_{\bar{n} i j}^{p} T_{p m \bar{k}}+\Omega_{\bar{n} i m}^{p} T_{j p \bar{k}}+\Omega_{\bar{n} i \bar{k}}^{\bar{p}} T_{j m \bar{p}} .
\end{aligned}
$$

Finally, using Lemma 2.1 we see

$$
\begin{aligned}
g^{m \bar{n}} \nabla_{\bar{n}}\left(\nabla_{j} T_{i m \bar{k}}-\nabla_{i} T_{j m \bar{k}}\right) & =g^{m \bar{n}} \nabla_{\bar{n}}\left(\nabla_{m} T_{i j \bar{k}}+T_{j i}^{p} T_{m p \bar{k}}+T_{m j}^{p} T_{i p \bar{k}}+T_{i m}^{p} T_{j p \bar{k}}\right) \\
& =\Delta T_{i j \bar{k}}+g^{m \bar{n}} \nabla_{\bar{n}}\left(T_{j i}^{p} T_{m p \bar{k}}+T_{m j}^{p} T_{i p \bar{k}}+T_{i m}^{p} T_{j p \bar{k}}\right) .
\end{aligned}
$$

Combining these calculations yields

$$
\begin{aligned}
\frac{\partial}{\partial t} T_{i j \bar{k}}= & \Delta T_{i j \bar{k}}+g^{m \bar{n}} \nabla_{\bar{n}}\left(T_{j i}^{p} T_{m p \bar{k}}+T_{m j}^{p} T_{i p \bar{k}}+T_{i m}^{p} T_{j p \bar{k}}\right) \\
& +g^{m \bar{n}}\left[\Omega_{\bar{n} j i}^{p} T_{p m \bar{k}}+\Omega_{\bar{n} j m}^{p} T_{i p \bar{k}}+\Omega_{\bar{n} j \bar{k}}^{\bar{p}} T_{i m \bar{p}}\right. \\
& \left.-\Omega_{\bar{n} i j}^{p} T_{p m \bar{k}}-\Omega_{\bar{n} i m}^{p} T_{j p \bar{k}}-\Omega_{\bar{n} i \bar{k}}^{\bar{p}} T_{j m \bar{p}}-\Omega_{p \bar{n} m \bar{k}} T_{j i}^{p}\right]-T_{i j}^{p} S_{p \bar{k}}
\end{aligned}
$$

Using the Bianchi identity we can simplify

$$
g^{m \bar{n}}\left[\Omega_{\bar{n} j i}^{p} T_{p m \bar{k}}-\Omega_{\bar{n} i j}^{p} T_{p m \bar{k}}\right]=g^{m \bar{n}}\left[\Omega_{\bar{n} j i}^{p}+\Omega_{i \bar{n} j}^{p}\right] T_{p m \bar{k}}=g^{m \bar{n}} \nabla_{\bar{n}} T_{j i}^{p} T_{p m \bar{k}} .
$$

Plugging this in yields

$$
\begin{aligned}
\frac{\partial}{\partial t} T_{i j \bar{k}}= & \Delta T_{i j \bar{k}}+g^{m \bar{n}}\left[T_{j i}^{p} \nabla_{\bar{n}} T_{m p \bar{k}}+\nabla_{\bar{n}} T_{m j}^{p} T_{i p \bar{k}}+T_{m j}^{p} \nabla_{\bar{n}} T_{i p \bar{k}}\right. \\
& \left.+\nabla_{\bar{n}} T_{i m}^{p} T_{j p \bar{k}}+T_{i m}^{p} \nabla_{\bar{n}} T_{j p \bar{k}}\right] \\
& +g^{m \bar{n}}\left[\Omega_{\bar{n} j m}^{p} T_{i p \bar{k}}+\Omega_{\bar{n} j \bar{k}}^{\bar{p}} T_{i m \bar{p}}-\Omega_{\bar{n} i m}^{p} T_{j p \bar{k}}-\Omega_{\bar{n} i \bar{k}}^{\bar{p}} T_{j m \bar{p}}-\Omega_{p \bar{n} m \bar{k}} T_{j i}^{p}\right] \\
& -T_{i j}^{p} S_{p \bar{k}}
\end{aligned}
$$

Combining this with the terms from $Q$ gives the result. 


\section{Higher derivative estimates}

In this section we will prove derivative estimates for HCF. It will be most convenient to phrase these results in terms of the curvature of the Chern connection. All of the calculations below will be done in canonical coordinates at a fixed point. In particular, in these coordinates any first derivative of $g$ can be expressed in terms of the torsion $T$, and any second derivative can be expressed in terms of a sum of curvature and torsion.

Lemma 7.1. Given $\left(M^{2 n}, g(t), J\right)$ a solution to $H C F$ we have

$$
\begin{aligned}
\frac{\partial}{\partial t} \nabla^{k} \Omega= & \Delta \nabla^{k} \Omega+\sum_{j=0}^{k} \nabla^{j} T * \nabla^{k+1-j} \Omega+\sum_{j=0}^{k} \nabla^{j} \Omega * \nabla^{k-j} \Omega \\
& +\sum_{j=0}^{k} \sum_{l=0}^{j} \nabla^{l} T * \nabla^{j-l} T * \nabla^{k-j} \Omega+\sum_{j=0}^{k+2} \nabla^{j} T * \nabla^{k+2-j} T .
\end{aligned}
$$

Proof. The case $k=0$ is covered by Lemma 6.1. We directly compute

$$
\begin{aligned}
\frac{\partial}{\partial t} \nabla^{k} \Omega= & \frac{\partial}{\partial t}(\partial+\Gamma) *(\partial+\Gamma) * \cdots *(\partial+\Gamma) \Omega \\
= & \nabla^{k}\left(\frac{\partial}{\partial t} \Omega\right)+\left(\frac{\partial}{\partial t} \Gamma\right) *(\partial+\Gamma) * \cdots *(\partial+\Gamma) \Omega \\
& +(\partial+\Gamma) *\left(\frac{\partial}{\partial t} \Gamma\right) * \cdots *(\partial+\Gamma) \Omega+\cdots \\
& +(\partial+\Gamma) * \cdots *(\partial+\Gamma) *\left(\frac{\partial}{\partial t} \Gamma\right) \Omega
\end{aligned}
$$

We apply Lemma 10.1 to see that

$$
\frac{\partial}{\partial t} \Gamma=\nabla\left(\Omega+T^{* 2}\right)=\nabla \Omega+T * \nabla T .
$$

Plugging this in yields

$$
\begin{aligned}
\frac{\partial}{\partial t} \nabla^{k} \Omega= & \nabla^{k}\left(\Delta \Omega+T * \nabla \Omega+\Omega^{* 2}+\Omega * T^{* 2}+\nabla^{2} Q\right) \\
& +\sum_{j=0}^{k-1} \nabla^{j}(\nabla \Omega+T * \nabla T) * \nabla^{k-1-j} \Omega \\
= & \Delta \nabla^{k} \Omega+\sum_{j=0}^{k} \nabla^{j} T * \nabla^{k+1-j} \Omega+\sum_{j=0}^{k} \nabla^{j} \Omega * \nabla^{k-j} \Omega \\
& +\sum_{j=0}^{k} \sum_{l=0}^{j} \nabla^{l} T * \nabla^{j-l} T * \nabla^{k-j} \Omega+\sum_{j=0}^{k+2} \nabla^{j} T * \nabla^{k+2-j} T .
\end{aligned}
$$


Lemma 7.2. Given $\left(M^{2 n}, g(t), J\right)$ a solution to HCF we have

$$
\begin{aligned}
\frac{\partial}{\partial t} \nabla^{k} T= & \Delta \nabla^{k} T+\sum_{j=0}^{k+1} \nabla^{j} T * \nabla^{k+1-j} T+\sum_{j=0}^{k} \nabla^{j} T * \nabla^{k-j} \Omega \\
& +\sum_{j=0}^{k-1} \sum_{l=0}^{j} \nabla^{l} T * \nabla^{j-l+1} T * \nabla^{k-1-j} T
\end{aligned}
$$

Proof. The case $k=0$ is covered by Lemma 6.2. We directly compute

$$
\begin{aligned}
\frac{\partial}{\partial t} \nabla^{k} T= & \frac{\partial}{\partial t}(\partial+\Gamma) * \cdots *(\partial+\Gamma) T \\
= & \nabla^{k}\left(\frac{\partial}{\partial t} T\right)+\left(\frac{\partial}{\partial t} \Gamma\right) *(\partial+\Gamma) * \cdots *(\partial+\Gamma) T \\
& +(\partial+\Gamma) *\left(\frac{\partial}{\partial t} \Gamma\right) * \cdots *(\partial+\Gamma) T+\cdots \\
& +(\partial+\Gamma) * \cdots *(\partial+\Gamma) *\left(\frac{\partial}{\partial t} \Gamma\right) T .
\end{aligned}
$$

Again we apply Lemma 10.1 to obtain

$$
\begin{aligned}
= & \nabla^{k}(\Delta T+\nabla T * T+\Omega * T)+\sum_{j=0}^{k-1} \nabla^{j}(\nabla \Omega+T * \nabla T) * \nabla^{k-1-j} T \\
= & \Delta \nabla^{k} T+\sum_{j=0}^{k+1} \nabla^{j} T * \nabla^{k+1-j} T+\sum_{j=0}^{k} \nabla^{j} T * \nabla^{k-j} \Omega \\
& +\sum_{j=0}^{k-1} \sum_{l=0}^{j} \nabla^{l} T * \nabla^{j-l+1} T * \nabla^{k-1-j} T .
\end{aligned}
$$

Theorem 7.3. Let $\left(M^{2 n}, g(t), J\right)$ be a solution to HCF for which the maximum principle holds. Then for each $\alpha>0$ and every $m \in \mathbb{N}$ there exists a constant $C_{m}$ depending only on $m, n$ and $\max \{\alpha, 1\}$ such that if

$$
|\Omega|_{C^{0}\left(g_{t}\right)} \leq K, \quad|\nabla T|_{C^{0}\left(g_{t}\right)} \leq K, \quad|T|_{C^{0}\left(g_{t}\right)}^{2} \leq K
$$

for all $x \in M$ and $t \in[0, \alpha / K]$, then

$$
\left|\nabla^{m} \Omega\right|_{C^{0}\left(g_{t}\right)} \leq \frac{C_{m} K}{t^{m / 2}}, \quad\left|\nabla^{m+1} T\right|_{C^{0}\left(g_{t}\right)} \leq \frac{C_{m} K}{t^{m / 2}}
$$

for all $x \in M$ and $t \in(0, \alpha / K]$. 
Proof. Our proof is by induction on $m$. First consider $m=1$. The following evolution equation for $|\Omega|^{2}$ follows from Lemma 7.1 .

$$
\begin{aligned}
\frac{\partial}{\partial t}|\Omega|^{2}= & \Delta|\Omega|^{2}-2|\nabla \Omega|^{2}+T * \nabla \Omega * \Omega+\Omega^{* 3}+T^{* 2} * \Omega^{* 2} \\
& +\nabla T * \nabla T * \Omega+T * \nabla^{2} T * \Omega \\
\leq & \Delta|\Omega|^{2}-|\nabla \Omega|^{2}+\frac{1}{2}\left|\nabla^{2} T\right|^{2}+C K^{3} .
\end{aligned}
$$

In the last line we applied the assumed bound on curvature in the time interval $[0, \alpha / K]$, and in what follows below $C$ will denote a generic constant depending only on the dimension. Also Lemma 7.1 implies

$$
\begin{aligned}
\frac{\partial}{\partial t}|\nabla \Omega|^{2}= & \Delta|\nabla \Omega|^{2}-2\left|\nabla^{2} \Omega\right|^{2}+T * \nabla^{2} \Omega * \nabla \Omega \\
& +\Omega * \nabla \Omega^{* 2}+\nabla T * \nabla \Omega^{* 2}+T^{* 2} * \nabla \Omega^{* 2} \\
& +T * \nabla T * \Omega * \nabla \Omega+T * \nabla^{3} T * \nabla \Omega+\nabla^{2} T * \nabla T * \nabla \Omega \\
\leq & \Delta|\nabla \Omega|^{2}-\left|\nabla^{2} \Omega\right|^{2}+\frac{1}{2}\left|\nabla^{3} T\right|^{2}+C K\left(|\nabla \Omega|^{2}+\left|\nabla^{2} T\right|^{2}\right)+C K^{4} .
\end{aligned}
$$

Also from Lemma 7.2 we conclude

$$
\begin{aligned}
\frac{\partial}{\partial t}|\nabla T|^{2}= & \Delta|\nabla T|^{2}-2\left|\nabla^{2} T\right|^{2}+T * \nabla^{2} T * \nabla T+\nabla T^{* 3} \\
& +T * \nabla \Omega * \nabla T+\nabla T * \Omega+T^{* 2} * \nabla T^{* 2} \\
\leq & \Delta|\nabla T|^{2}-\left|\nabla^{2} T\right|^{2}+\frac{1}{2}|\nabla \Omega|^{2}+C K^{3}
\end{aligned}
$$

and

$$
\begin{aligned}
\frac{\partial}{\partial t}\left|\nabla^{2} T\right|^{2}= & \Delta\left|\nabla^{2} T\right|^{2}-2\left|\nabla^{3} T\right|^{2}+T * \nabla^{3} T * \nabla^{2} T+\nabla T * \nabla^{2} T * \nabla^{2} T \\
& +T * \nabla^{2} \Omega * \nabla^{2} T+\nabla T * \nabla \Omega * \nabla^{2} T+\nabla^{2} T^{* 2} * \Omega \\
& +T * \nabla T^{* 2} * \nabla^{2} T+T^{* 2} * \nabla^{2} T^{* 2} \\
\leq & \Delta\left|\nabla^{2} T\right|^{2}-\left|\nabla^{3} T\right|^{2}+\frac{1}{2}\left|\nabla^{2} \Omega\right|^{2}+C K\left(|\nabla \Omega|^{2}+\left|\nabla^{2} T\right|^{2}\right)+C K^{4} .
\end{aligned}
$$

Consider the function

$$
F(x, t):=t\left(|\nabla \Omega|^{2}+\left|\nabla^{2} T\right|^{2}\right)+\beta\left(|\Omega|^{2}+|\nabla T|^{2}\right)
$$

where $\beta$ is a constant to be chosen below. Putting together (16)-19 and using $t \in$ $[0, \alpha / K]$ gives

$$
\begin{aligned}
\frac{\partial}{\partial t} F \leq & \Delta F-\frac{t}{2}\left(\left|\nabla^{2} \Omega\right|^{2}+\left|\nabla^{3} T\right|^{2}\right)+(t C K-\beta / 2)\left(|\nabla \Omega|^{2}+\left|\nabla^{2} T\right|^{2}\right) \\
& +C\left(t K^{4}+\beta K^{3}\right) \\
\leq & \Delta F+(C \alpha-\beta / 2)\left(|\nabla \Omega|^{2}+\left|\nabla^{2} T\right|^{2}\right)+C K^{3}(\alpha+\beta) .
\end{aligned}
$$


Choosing $\beta$ large with respect to dimensional constants and $\max \{\alpha, 1\}$ yields

$$
\frac{\partial}{\partial t} F \leq C \beta K^{3}
$$

Using that $F(0) \leq \beta K^{2}$ and applying the maximum principle gives

$$
\sup _{x \in M} F(x, t) \leq \beta K^{2}+C \beta K^{3} t \leq(1+C \alpha) \beta K^{2} \leq C_{1}^{2} K^{2}
$$

where again $C_{1}$ depends only on $n$ and $\max \{\alpha, 1\}$. Thus

$$
|\nabla \Omega|+\left|\nabla^{2} T\right| \leq \sqrt{\frac{F}{t}} \leq \frac{C_{1} K}{t^{1 / 2}}
$$

for all $x \in M$ and $t \in(0, \alpha / K]$. This completes the case $m=1$.

For the induction step we first deduce from Lemma 7.1 the evolution equation

$$
\begin{aligned}
\frac{\partial}{\partial t}\left|\nabla^{k} \Omega\right|^{2}= & \Delta\left|\nabla^{k} \Omega\right|^{2}-2\left|\nabla^{k+1} \Omega\right|^{2}+\sum_{j=0}^{k} \nabla^{j} T * \nabla^{k+1-j} \Omega * \nabla^{k} \Omega \\
& +\sum_{j=0}^{k} \nabla^{j} \Omega * \nabla^{k-j} \Omega * \nabla^{k} \Omega+\sum_{j=0}^{k} \sum_{l=0}^{j} \nabla^{l} T * \nabla^{j-l} T * \nabla^{k-j} \Omega * \nabla^{k} \Omega \\
& +\sum_{j=0}^{k+2} \nabla^{j} T * \nabla^{k+2-j} T * \nabla^{k} \Omega .
\end{aligned}
$$

We address the first sum in the above equation. We first make the bound

$$
\begin{aligned}
T * \nabla^{k+1} \Omega * \nabla^{k} \Omega & \leq C|T|\left|\nabla^{k+1} \Omega\right|\left|\nabla^{k} \Omega\right| \leq \frac{1}{2}\left|\nabla^{k+1} \Omega\right|^{2}+C|T|^{2}\left|\nabla^{k} \Omega\right|^{2} \\
& \leq \frac{1}{2}\left|\nabla^{k+1} \Omega\right|^{2}+C K\left|\nabla^{k} \Omega\right|^{2}
\end{aligned}
$$

Also we have

$$
\nabla T * \nabla^{k} \Omega * \nabla^{k} \Omega \leq C|\nabla T|\left|\nabla^{k} \Omega\right|^{2} \leq C K\left|\nabla^{k} \Omega\right|^{2} .
$$

For the rest of the summand we bound, for $j>0$,

$$
\begin{aligned}
\nabla^{j} T * \nabla^{k+1-j} \Omega * \nabla^{k} \Omega & \leq C\left|\nabla^{j} T\right|\left|\nabla^{k+1-j} \Omega\right|\left|\nabla^{k} \Omega\right| \\
& \leq C \frac{K}{t^{(j-1) / 2}} \frac{K}{t^{(k+1-j) / 2}}\left|\nabla^{k} \Omega\right| \leq C K\left|\nabla^{k} \Omega\right|^{2}+C \frac{K^{3}}{t^{k}} .
\end{aligned}
$$

A similar calculation yields a bound

$$
\begin{aligned}
\nabla^{j} \Omega * \nabla^{k-j} \Omega * \nabla^{k} \Omega & \leq C\left|\nabla^{j} \Omega\right|\left|\nabla^{k-j} \Omega\right|\left|\nabla^{k} \Omega\right| \\
& \leq C \frac{K}{t^{j / 2}} \frac{K}{t^{(k-j) / 2}}\left|\nabla^{k} \Omega\right| \leq C K\left|\nabla^{k} \Omega\right|^{2}+C \frac{K^{3}}{t^{k}}
\end{aligned}
$$


Next we bound using the inequality $K \leq C / t$ :

$$
\begin{aligned}
\nabla^{l} T * \nabla^{j-l} T * \nabla^{k-j} \Omega * \nabla^{k} \Omega & \leq C\left|\nabla^{l} T\right|\left|\nabla^{j-l} T\right|\left|\nabla^{k-j} \Omega\right|\left|\nabla^{k} \Omega\right| \\
& \leq C \frac{K}{t^{(l-1) / 2}} \frac{K}{t^{(j-l-1) / 2}} \frac{K}{t^{(k-j) / 2}}\left|\nabla^{k} \Omega\right| \\
& \leq C \frac{K^{3}}{t^{(k-2) / 2}}\left|\nabla^{k} \Omega\right| \leq C \frac{K^{2}}{t^{(k-1) / 2}}\left|\nabla^{k} \Omega\right| \\
& \leq C K\left|\nabla^{k} \Omega\right|^{2}+C \frac{K^{3}}{t^{k}} .
\end{aligned}
$$

Finally we bound the last summand. First, for $j=0$ we have

$$
T * \nabla^{k+2} T * \nabla^{k} \Omega \leq \frac{1}{2}\left|\nabla^{k+2} T\right|^{2}+C K\left|\nabla^{k} \Omega\right|^{2} .
$$

For the general term, estimates as above yield

$$
\nabla^{j} T * \nabla^{k+2-j} T * \nabla^{k} \Omega \leq C K\left(\left|\nabla^{k} \Omega\right|^{2}+\left|\nabla^{k+1} T\right|^{2}+\frac{K^{2}}{t^{k}}\right) .
$$

Using (21)-27) we conclude

$$
\begin{aligned}
\frac{\partial}{\partial t}\left|\nabla^{k} \Omega\right|^{2} \leq & \Delta\left|\nabla^{k} \Omega\right|^{2}-\left|\nabla^{k+1} \Omega\right|^{2}+\frac{1}{2}\left|\nabla^{k+2} T\right|^{2} \\
& +C K\left(\left|\nabla^{k} \Omega\right|^{2}+\left|\nabla^{k+1} T\right|^{2}+\frac{K^{2}}{t^{k}}\right) .
\end{aligned}
$$

Furthermore, using completely analogous bounds one can conclude

$$
\begin{aligned}
\frac{\partial}{\partial t}\left|\nabla^{k+1} T\right|^{2} \leq & \Delta\left|\nabla^{k+1} T\right|^{2}-\left|\nabla^{k+2} T\right|^{2}+\frac{1}{2}\left|\nabla^{k+1} \Omega\right|^{2} \\
& +C K\left(\left|\nabla^{k} \Omega\right|^{2}+\left|\nabla^{k+1} T\right|^{2}+\frac{K^{2}}{t^{k}}\right) .
\end{aligned}
$$

Together these yield, if we set $H_{k}=\left|\nabla^{k} \Omega\right|^{2}+\left|\nabla^{k+1} T\right|^{2}$,

$$
\frac{\partial}{\partial t} H_{k} \leq \Delta H_{k}-\frac{1}{2} H_{k+1}+C K H_{k}+\frac{K^{3}}{t^{k}} .
$$

This bound is sufficient to carry out the inductive step analogously to the step $k=1$. The details of this construction are found in [6, pp. 229-230].

Corollary 7.4. There exists a constant $c=c(n)$ such that given $\left(M^{2 n}, g, J\right)$ a complex manifold with Hermitian metric $g$, the solution to HCF with initial condition $g$ exists for $t \in\left[0, c(n) / \max \left\{|\Omega|_{C^{0}},|\nabla T|_{C^{0}},|T|_{C_{0}}^{2}\right\}\right]$. Moreover the solution exists on a maximal time interval $[0, \tau)$, and if $\tau<\infty$ then

$$
\limsup _{t \rightarrow \tau} \max \left\{|\Omega|_{C^{0}\left(g_{t}\right)},|\nabla T|_{C^{0}\left(g_{t}\right)},|T|_{C^{0}\left(g_{t}\right)}^{2}\right\}=\infty .
$$


Proof. This argument is standard. Using the evolution equations for $T, \nabla T$ and $\Omega$ it is easy to prove a "doubling-time" estimate for these quantities on the interval stated using the maximum principle. Once this is in place, the derivative estimates follow from Theorem 7.3 These yield bounds on the curvature and torsion and all covariant derivatives on the stated interval, which can be integrated in time to show smooth existence of the flow on that interval.

Finally, if one knows that the curvature, torsion and first covariant derivative of torsion are bounded up to a time $\tau<\infty$, one deduces from Theorem 7.3 uniform bounds on the derivatives of curvature and torsion on $[0, \tau]$. These bounds can be integrated in time to get $C^{k}$ bounds on the metric on this whole time interval, yielding smooth existence up to this time.

Note now that Theorem 1.1 is a consequence of Proposition 5.1, Theorem 7.3 and Corollary 7.4

\section{Stability}

In this section we prove dynamic stability of HCF near a Kähler-Einstein metric with negative or zero first Chern class. By examining the linearized deformation equation we know that Kähler-Einstein metrics are rigid in case $c_{1}(M)<0$. In the case $c_{1}(M)=0$, there can be nontrivial deformation of Kähler-Einstein metrics due to variation of Kähler class. There is a general technique for dealing with stability of evolution equations around integrable stationary points [5], [14], [15]. Given the discussion above, our problem falls squarely into the realm of these techniques, and so we adopt them. We note that since the $c_{1}(M)<0$ case is rigid, there may be an easier proof for this case, but in the interest of covering the most cases possible with a single proof we choose the more general technique.

Consider the volume-normalized HCF equation

$$
\frac{\partial}{\partial t} g=-S+Q+\frac{1}{n}\left(\int_{M} \operatorname{tr}_{g}(S-Q) d V\right) g=:-\mathcal{F}(g) .
$$

We compute the linearization of $\mathcal{F}$ at a Kähler-Einstein metric. Since the tensor $\mathcal{F}(g)$ is only defined for Hermitian metrics we obviously compute the variation of $\mathcal{F}(g)$ through a family of Hermitian metrics.

Proposition 8.1. Let $\left(M^{2 n}, J\right)$ be a complex manifold and suppose $g(a)$ is a one-parameter family of unit volume Hermitian metrics compatible with $J$ with

$$
\left.\frac{\partial}{\partial a} g(a)\right|_{a=0}=h .
$$

Moreover suppose $g(0)$ is Kähler-Einstein. Then

$$
\frac{\partial}{\partial a} \mathcal{F}(g)=\nabla^{*} \nabla h-\stackrel{\circ}{R}(h)
$$

where $\stackrel{\circ}{R}(h)_{k \bar{l}}=h^{i \bar{j}} R_{k \bar{j} i \bar{l}}$. 
Proof. Choose complex coordinates which are normal for $g(0)$ at a point $p \in M$. First we note that

$$
\left.\frac{\partial}{\partial a} T(a) * T(a)\right|_{a=0}=h * T(0) * T(0)+\left(\frac{\partial}{\partial a} T(a)\right) * T(0)=0
$$

since the metric $g(0)$ is Kähler and hence torsion-free. Now using Lemma 2.2 ,

$$
\begin{aligned}
\left.\frac{\partial}{\partial a} S_{j \bar{k}}\right|_{a=0} & =\left.\frac{\partial}{\partial a}\left(g(a)^{l \bar{m}}\left(-\partial_{l} \partial_{\bar{m}} g(a)_{j \bar{k}}+\partial g(a) * \bar{\partial} g(a)\right)\right)\right|_{a=0} \\
& =-h^{l \bar{m}} R_{l \bar{m} j \bar{k}}-g^{l \bar{m}} \partial_{l} \partial_{\bar{m}} h_{j \bar{k}} .
\end{aligned}
$$

Now $-h^{i \bar{j}} R_{i \bar{j} k \bar{l}}=-\stackrel{\circ}{R}(h)_{k \bar{l}}$ from the Bianchi identity using that the metric $g(0)$ is Kähler-Einstein. Next, we compute an expression for $\nabla^{*} \nabla h$ using complex coordinates:

$$
\begin{aligned}
\left(\nabla^{*} \nabla h\right)_{j \bar{k}} & =-g^{l \bar{m}} \nabla_{l} \nabla_{\bar{m}} h_{j \bar{k}}=-g^{l \bar{m}}\left(\partial_{l} \partial_{\bar{m}} h_{j \bar{k}}-\partial_{l} \Gamma_{\bar{m} \bar{k}}^{\bar{p}} h_{j \bar{p}}\right) \\
& =-g^{l \bar{m}} \partial_{l} \partial_{\bar{m}} h_{j \bar{k}}-R_{\bar{k}}^{\bar{m}} h_{j \bar{m}}=-g^{l \bar{m}} \partial_{l} \partial_{\bar{m}} h_{j \bar{k}}-\frac{1}{n} s h_{j \bar{k}}
\end{aligned}
$$

where $R=S$ is the Ricci tensor of the Kähler metric $g(0)$ and $s=r=\operatorname{tr}_{g} S$ is the scalar curvature. Next we compute using Lemma 10.7

$$
\begin{aligned}
\left.\frac{\partial}{\partial a}\left(\int_{M} \operatorname{tr}_{g} S d V\right)\right|_{a=0} & =\int_{M}\left(\langle h,-S\rangle+\operatorname{tr}_{g} h \operatorname{tr}_{g} S\right) d V=\int_{M}\left(1-\frac{1}{n}\right) \operatorname{tr}_{g} S \operatorname{tr}_{g} h d V \\
& =0
\end{aligned}
$$

where the last equality follows since $\operatorname{tr}_{g} S$ is the scalar curvature which is constant and $\int_{M} \operatorname{tr}_{g} h d V=0$ since the volume is fixed through $g(s)$. Thus

$$
\left.\frac{\partial}{\partial a} \frac{1}{n}\left(\int_{M} \operatorname{tr}_{g} S d V\right) g\right|_{a=0}=\frac{1}{n}\left(\int_{M} \operatorname{tr}_{g} S d V\right) h=\frac{1}{n} s h .
$$

Putting together these calculations yields the result.

Definition 8.2. Let $L=L\left(g_{0}\right)=\mathcal{D}_{g_{0}} \mathcal{F}$ be the linearization of $\mathcal{F}$ at a static metric $g_{0}$. We say that $g_{0}$ is linearly stable if $L \geq 0$.

Definition 8.3. A static metric $g_{0}$ is integrable if for any solution $h$ of the linearized equation

$$
\mathcal{D}_{g_{0}}(\mathcal{F}(g))(h)=0
$$

there exists a path $g(s), s \in(-\epsilon, \epsilon)$, of static metrics where $g(0)=g_{0}$ and

$$
\left.\frac{d}{d s}\right|_{s=0} g(s)=h .
$$

In particular this implies that the set of Hermitian metrics $g$ satisfying $\mathcal{F}(g) \equiv 0$ has a smooth manifold structure near $g_{0}$. 
We note that by the analysis of Koiso's Theorem it follows that Kähler-Einstein metrics with $c_{1}(M)<0$ or $c_{1}(M)=0$ are integrable. Indeed, any solution to the linearized deformation equation arises as the variation along a path of Kähler-Einstein metrics, which are static. This can be seen as follows: If $c_{1}(M)<0$, Kähler-Einstein metrics are linearly stable. If $c_{1}(M)=0$, any infinitesimal deformation of Kähler-Einstein metrics is given by Hermitian symmetric deformation of Einstein metrics which in turn correspond to $(1,1)$-forms; moreover, the eigenvalues $L$ are the eigenvalues of the operator

$$
\psi \mapsto \Delta_{d} \psi-\frac{1}{n} s \psi
$$

acting on $(1,1)$-forms $\psi([2$, p. 362]). If $s=0$, nonnegativity follows easily and the kernel of $L$ consists of harmonic $(1,1)$-forms which are simply variations of Kähler-Einstein metrics with vanishing scalar curvature due to the Calabi-Yau theorem. So Kähler-Einstein metrics are integrable in the case $c_{1}(M)=0$. We now proceed with the proof of Theorem 1.2.

Proof of Theorem 1.2. Let $\left(M, g_{0}, J\right)$ be a Kähler-Einstein manifold. Fix $h$ a symmetric two-tensor of type $(1,1)$ such that $|h|_{C^{\infty}}<\epsilon^{\prime}<\epsilon$ where $\epsilon^{\prime}$ and $\epsilon$ are small positive constants to be chosen later. We want to show that the solution to the equation

$$
\begin{aligned}
\frac{\partial}{\partial t} g & =-S+Q(T)+\frac{1}{n}\left(\int_{M} \operatorname{tr}_{g}(S-Q) d V\right) g, \\
g(0) & =g_{0}+h,
\end{aligned}
$$

exists for all time and converges for $\epsilon^{\prime}$ chosen small enough. Let $h(t)=g(t)-g_{0}$. First consider

$$
\begin{aligned}
\frac{\partial}{\partial t} h & =-S+Q+\frac{1}{n}\left(\int_{M} \operatorname{tr}_{g}(S-Q) d V\right) g \\
& =-\left(\mathcal{F}\left(g_{0}\right)+\mathcal{D} \mathcal{F}_{g_{0}}(h)+A\left(g_{0}, h\right)\right) \\
& =-\mathcal{D} \mathcal{F}_{g_{0}}(h)+A\left(g_{0}, h\right)=-L(h)+A\left(g_{0}, h\right)
\end{aligned}
$$

where $A$ represents the higher order terms in the approximation of $\mathcal{F}$ by $\mathcal{D} S_{g_{0}}(h)$. Specifically we have the bounds

$$
\left|A\left(g_{0}, h\right)\right|_{C^{k}} \leq C\left(|h|_{C^{k}}\left|\nabla^{2} h\right|_{C^{k-2}}+|\nabla h|_{C^{k-1}}^{2}\right)
$$

where the constant $C$ depends on bounds on the geometry of $g(t)$, which we are assuming is staying bounded along the flow anyway since $\left|g(t)-g_{0}\right|_{C^{k}}<\epsilon$. So, fix $T>0$ and a small $\epsilon>0$. We would like to show that for $\epsilon^{\prime}$ small enough as above our solution exists on $[0, T)$ and $|h(t)|_{C^{k}}<\epsilon$ on this interval. We start with an $L^{2}$ growth estimate.

Lemma 8.4. There exists a uniform (independent of $\epsilon, \epsilon^{\prime}, T$ ) constant $C$ such that if $|h|_{C^{k}}<\epsilon$ for all $t \in[0, T)$, we have

$$
\int_{M}|h(t)|^{2} d V_{g_{0}} \leq e^{C \epsilon t} \int_{M}\left|h_{0}\right|^{2} d V_{g_{0}} .
$$


Proof. Multiplying the final equation in (29) by $h$ and integrating over $M$ gives

$$
\frac{\partial}{\partial t} \int_{M}|h(t)|^{2} d V_{g_{0}} \leq \int_{M}(A * h) d V_{g_{0}}
$$

since $L$ is negative semidefinite. By straightforward bounds using integration by parts and the assumed $C^{k}$ bound on $h$ we are able to get the bound

$$
\left|\int_{M}(A * h) d V_{g_{0}}\right| \leq C \epsilon \int_{M}|h|^{2} d V_{g_{0}}
$$

where $C$ depends only on $g_{0}$. The result follows immediately.

Lemma 8.5. There exists $\epsilon^{\prime}=\epsilon^{\prime}(T, n) \ll \epsilon$ such that if $\left|h_{0}\right|_{C^{\infty}}<\epsilon^{\prime}$, then the solution $h(t)$ exists on $[0, T)$ with $|h(t)|_{C^{k}}<\epsilon$ for all $t \in[0, T)$.

Proof. We use standard parabolic regularity theory. First we rewrite the evolution equation for $h$ as

$$
\frac{\partial}{\partial t} h=\Delta h+\operatorname{Rm}(h)+A\left(g_{0}, h\right)
$$

Fix a time $\tau<T$. We will first get an estimate for $\int_{0}^{\tau} \int_{M}|\nabla h|^{2} d V_{g_{0}} d t$. Take the inner product of (31) with $h$ and integrate over $M$ to get

$$
\begin{aligned}
\frac{1}{2} \frac{\partial}{\partial t} \int_{M}|h|^{2} & =-\int_{M}|\nabla h|^{2}+\int_{M} \mathrm{Rm} * h^{* 2}+\int_{M} \nabla^{2} h * h^{* 2}+h * \nabla h^{* 2} \\
& \leq-\int_{M}|\nabla h|^{2}+\theta \int_{M}|\nabla h|^{2}+C(\theta) \int_{M}|h|^{2} \\
& \leq-\frac{1}{2} \int_{M}|\nabla h|^{2}+C(\theta) \int_{M}|h|^{2} .
\end{aligned}
$$

Using this bound and integrating over time we conclude that

$$
\frac{1}{2} \int_{0}^{\tau} \int_{M}|\nabla h|^{2} \leq \frac{1}{2} \int_{M}\left|h_{0}\right|^{2}+C(\theta) \tau \sup _{[0, \tau)} \int_{M}|h(t)|^{2} .
$$

Using Lemma 8.4 we see that $\int_{0}^{\tau} \int_{M}|\nabla h|^{2}$ can be made very small, in particular bounded uniformly in terms of $\epsilon^{\prime}$. We now show how to get estimates on $\int_{0}^{\tau} \int_{M}\left|\nabla^{k} h\right|^{2}$ for all $k>0$ in terms of the small constant $\epsilon^{\prime}$. Consider

$$
\begin{aligned}
\frac{1}{2} \frac{\partial}{\partial t} \int_{M}|\nabla h|^{2}= & \int_{M}\left\langle\nabla\left(\Delta h+\mathrm{Rm}(h)+A\left(g_{0}, h\right)\right), \nabla h\right\rangle \\
& +\int_{M} \nabla^{2} h * \nabla h * \nabla h+h * \nabla h^{* 2} \\
\leq & -\int_{M}\left|\nabla^{2} h\right|^{2}+C \int_{M}|\mathrm{Rm}||\nabla \mathrm{h}|^{2} \\
& +\theta \int_{M}\left|\nabla^{2} h\right|^{2}+C(\theta) \int_{M}|\nabla h|^{4}+C \epsilon^{\prime} \int_{M}|\nabla h|^{2} \\
\leq & -\frac{1}{2} \int_{M}\left|\nabla^{2} h\right|^{2}+C \int_{M}|\nabla h|^{2} .
\end{aligned}
$$


This implies the bound

$$
\frac{1}{2} \int_{0}^{\tau} \int_{M}\left|\nabla^{2} h\right|^{2} \leq \frac{1}{2} \int_{M}\left|\nabla h_{0}\right|^{2}+C \int_{0}^{\tau} \int_{M}|\nabla h|^{2} \leq C \epsilon^{\prime} .
$$

Continuing in this fashion we can induct to get a bound of the above form for all covariant derivatives of $h$. Note that for instance we can now bound

$$
\int_{0}^{\tau} \int_{M}\left|\frac{\partial}{\partial t} h\right|^{2} \leq C\left(\int_{0}^{\tau} \int_{M}\left|\nabla^{2} h\right|^{2}+\int_{0}^{\tau} \int_{M}|h|^{2}\right) \leq C \epsilon^{\prime} .
$$

It is clear that we can in fact get bounds of the form

$$
\int_{0}^{\tau} \int_{M}\left|\frac{\partial^{p}}{\partial t^{p}} \nabla^{q} h\right|^{2} \leq C \epsilon^{\prime}
$$

for all $p, q>0$. One can now apply the Sobolev inequality (with respect to $g_{0}$ ) to deduce $C^{k}$ bounds on $h$ in terms of $\epsilon^{\prime}$. These bounds will hold over any time interval where the $L^{2}$ norm of $h$ is still small. Since this time can be made arbitrarily large with small choice of $\epsilon^{\prime}$ by Lemma 8.4 , the result follows.

We now improve these estimates to include $L^{2}$ decay of $h$, which will ultimately yield the stated long-time existence and convergence. Say $T$ is a maximal time such that $|h|_{k}<\epsilon$ on $[0, T)$. Divide the interval $[0, T)$ into intervals of length $\tau$ and let $N$ be the integer such that $N \tau<T<(N+1) \tau$. Let $I_{j}=[j \tau,(j+1) \tau]$. On $M_{j}:=M \times I_{j}$ define a norm

$$
\|f\|_{M_{j}}:=\int_{j \tau}^{(j+1) \tau}\|f(t)\|_{L^{2}\left(g_{0}\right)} d t
$$

which defines an inner product in the usual way. Let $\pi^{j}$ denote the orthogonal projection onto $\operatorname{ker}(\partial / \partial t+L)$ with respect to $\|f\|_{M_{j}}$. Since $L$ is positive semidefinite we see that $\pi$ has no positive eigenvalues, but there is still the lingering question of zero eigenvalues. This is where the integrability property comes in. Let $\pi_{0}^{j}(h)$ denote the radial component, i.e. the kernel of $L$. We will show using integrability that there exists a stationary solution $g_{j}$ on $M_{j}$ such that $\pi_{0}^{j}\left(g(t)-g_{j}\right)$ is very small compared to $g(t)-g_{j}$. This will allow us to deduce the $L^{2}$ decay of $h$ and then to conclude convergence.

Lemma 8.6. Given $\alpha>0$ there exists $\delta=\delta(n, \tau)$ such that if $\sup _{\left[\tau_{0}, \tau_{0}+\tau\right]}|h(t)|_{C^{k}}<\delta$ then there exists a Kähler-Einstein metric $g_{1}$ such that

$$
\left|\pi_{0}^{j}\left(g-g_{1}\right)\right| \leq \alpha\left(t-\tau_{0}\right)\left|g-g_{1}\right|
$$

and

$$
\left|g_{1}-g_{0}\right|_{C^{k}} \leq C \sup _{I}\left|g-g_{0}\right|_{C^{k}} .
$$


Proof. Recall from the discussion above that the set of metrics $g$ near $g_{0}$ satisfying $\mathcal{F}(g)=0$, call it $\mathcal{U}$, has a natural smooth manifold structure. The tangent space to $\mathcal{U}$ is given by the kernel of $L$, call it $\mathcal{K}$, which is finite-dimensional since $L$ is elliptic. Let $\left\{B_{i}\right\}$ be a basis for $\mathcal{K}$ orthonormal with respect to the $L^{2}$ norm induced by $g_{0}$. Also using ellipticity, we get a system of eigenvectors $\left\{E_{\lambda}\right\}$ for $L$ orthonormal with respect to the $L^{2}$ inner product above. We see that there exist constants $r_{\lambda}$ such that $C_{\lambda}=r_{\lambda} E_{\lambda} e^{\lambda t}$ is a basis for $\operatorname{ker}(\partial / \partial t+L)$ which is orthonormal with respect to the inner product corresponding to 33 .

Define the map $\Psi: \mathcal{U} \rightarrow \mathcal{K}$ by $\Psi(g)=\sum_{i}\left\langle g, B_{i}\right\rangle>B_{i}$. A simple calculation using the bases described above shows that for $g_{1} \in \mathcal{U}, \Psi\left(g_{1}\right)=\Psi\left(\pi^{j}\left(g_{1}\right)\right)=\pi_{0}^{j}\left(g_{1}\right)$. Also it is easy to see that the differential of $\Psi$ at $g_{0}$ is the identity map, so we can apply the inverse function theorem. Fix the time $\tau_{0} \in I_{j}$. If $\left|g(t)-g_{0}\right|_{k}$ is small enough, then in particular $\pi_{0}^{j}\left(g\left(\tau_{0}\right)-g_{0}\right)=\pi_{0}^{j}\left(g\left(\tau_{0}\right)\right)$ can be made small, so that by the argument above there exists $g_{1} \in \mathcal{U}$ such that

$$
\Psi\left(g_{1}\right)=\pi_{0}^{j}\left(g\left(\tau_{0}\right)\right) .
$$

Thus in particular using the above equalities we have $\pi_{0}^{j}\left(g_{1}-g\left(\tau_{0}\right)\right)=0$. Using the evolution equations satisfied by $g$ and $g_{1}$ it is clear that one has estimate (34). Also note that $g_{1}=\Psi^{-1}\left(\left(\pi^{j} g\right)_{0}\right)$ and $g_{0}=\Psi^{-1}\left(\left(\pi^{j} g_{0}\right)_{0}\right)$, thus using our bound from the inverse function theorem we get

$$
\left\|g_{1}-g_{0}\right\|_{M_{j}} \leq C\left\|\pi^{j}\left(g-g_{0}\right)\right\|_{M_{j}}
$$

and again using that these are all solutions of the same parabolic equation, we can get the bound

$$
\left|g_{1}-g_{0}\right|_{C^{k}} \leq C \sup _{I_{j}}\left|g-g_{0}\right|_{C^{k}}
$$

Lemma 8.7. Let $I=\left[\tau_{0}, \tau_{0}+\tau\right]$ and take $g_{1}$ as in Lemma 8.6 Then there exists $\epsilon>0$ depending only on $g_{0}$ such that if $\left|h_{1}(0)\right|_{C^{k}}<\epsilon$ where $h_{1}=g-g_{1}$ then

$$
\sup _{\left[\tau_{0}+\tau / 2, \tau_{0}+\tau\right]} \int_{M}\left|g-g_{1}\right|^{2} d V_{g_{0}} \leq e^{-\tau \lambda / 2} \sup _{\left[\tau_{0}, \tau_{0}+\tau / 2\right]} \int_{M}\left|g-g_{1}\right|^{2} d V_{g_{0}}
$$

where $\lambda=\min \left\{\lambda_{i}: \lambda_{i}\right.$ is an eigenvalue of $\left.L, \lambda_{i} \neq 0\right\}>0$.

Proof. Let $h_{1}(t)=g(t)-g_{1}$. If $\left|h_{1}(0)\right|_{C^{k}}<\epsilon$, a calculation like that in Lemma 8.4 combined with the bound on $\pi_{0}\left(h_{1}(t)\right)$ shows that

$$
\begin{aligned}
\frac{d}{d t} \int_{M}\left|h_{1}\right|^{2} & =\int_{M}\left\langle 2 L h_{1}, h_{1}\right\rangle d V_{g_{0}}+\int_{M} A\left(h_{1}, g_{0}\right) * h_{1} d V_{g_{0}} \\
& \leq-2 \lambda \int_{M}\left|h_{1}-\pi_{0}^{j}\left(h_{1}\right)\right|^{2} d V_{g_{0}}+C \epsilon \int_{M}\left|h_{1}\right|^{2} d V_{g_{0}} \\
& \leq\left(-\frac{3}{2} \lambda+C \epsilon\right) \int_{M}\left|h_{1}\right|^{2} \leq-\lambda \int_{M}\left|h_{1}\right|^{2}
\end{aligned}
$$

as long as $\epsilon<\lambda / C$. Thus $\int_{M}\left|h_{1}(t)\right|^{2} d V_{g_{0}} \leq e^{-\lambda(t-\tau)} \int_{M}\left|h_{1}(\tau)\right|^{2} d V_{g_{0}}$, from which the claim follows immediately. 
We will need one more lemma, which roughly says that if a solution to 28 is decaying at a certain rate at a particular time then it decayed at that rate earlier in time. This lemma is inspired by Lemma 5.31 in [5], and the proof is the same.

Lemma 8.8. There exists a constant $v(n, \tau)>0$ with the following property. Let $h$ be a symmetric two-tensor satisfying the equation

$$
\frac{\partial}{\partial t} h=-L h+A\left(g_{0}, h\right)
$$

and

$$
\sup _{\left[\tau_{0}, \tau_{0}+\tau\right]}|h|_{C^{k}}<v \quad \text { and } \quad\left|\pi_{0}(h)\right| \leq \alpha\left(t-\tau_{0}\right)|h|
$$

where $\pi_{0}$ is the projection onto the kernel of $\partial / \partial t+L$ restricted to the interval $\left[\tau_{0}-\tau / 2, \tau_{0}+\tau\right]$. Then if

$$
\sup _{\left[\tau_{0}+\tau / 2, \tau_{0}+\tau\right]} \int_{M}|h|^{2} d V_{g_{0}} \leq e^{-\tau \lambda / 2} \sup _{\left[\tau_{0}, \tau_{0}+\tau / 2\right]} \int_{M}|h|^{2} d V_{g_{0}}
$$

then

$$
\sup _{\left[\tau_{0}, \tau_{0}+\tau / 2\right]} \int_{M}|h|^{2} d V_{g_{0}} \leq e^{-\tau \lambda / 2} \sup _{\left[\tau_{0}-\tau / 2, \tau_{0}\right]} \int_{M}|h|^{2} d V_{g_{0}} .
$$

Proof. First note that the analogous claim where $h$ satisfies the linear equation $(\partial / \partial t+L) h$ $=0$ and $\pi_{0}(h)=0$ is obvious since $L$ is positive semidefinite and by definition $\pi(h)_{0}=0$. In fact there is decay at the rate $\lambda$ as opposed to the $\lambda / 2$ in the statement above. So, if the claim were false, then for a sequence $v_{i} \rightarrow 0$ we would have $h_{i}$ satisfying the hypothesis but not the conclusion with the bound $\left|h_{i}\right|_{C^{k}}<v_{i}$. By standard compactness arguments we can parabolically rescale $h_{i}$ and extract a subsequence converging to $h_{\infty}$, which satisfies the initial decay hypothesis but not the conclusion. Moreover, given that $A$ is quadratic in $h$, it is clear that this $h_{\infty}$ satisfies the linear equation $(\partial / \partial t+L) h=0$ and $\pi_{0}(h)=0$, contradicting the above.

We now proceed with the main proof. Suppose the maximal existence time satisfies $T<\infty$, and subdivide [0,T] into $N$ intervals of length $\tau$ labeled $I_{j}$ as above. For fixed $j$, let $g_{j}$ be the metric such that $\pi_{0}^{j}\left(g(t)-g_{j}\right)=0$ on $I_{j}$ given by Lemma 8.6 Define $h_{j}:=g(t)-g_{j}$. By Lemma 8.7 and parabolic regularity we have

$$
\sup _{[(j+1 / 2) \tau,(j+1) \tau]}\left|h_{j}\right| \leq C e^{-\tau \lambda / 2} \sup _{[j \tau,(j+1 / 2) \tau]}\left|h_{j}\right| .
$$

We can apply Lemma 8.8 inductively to deduce

$$
\sup _{[j \tau,(j+1) \tau]}\left|h_{j}\right| \leq e^{-\lambda \tau(j-1)} \sup _{[0, \tau / 2]}\left|h_{j}\right| .
$$

This allows us to conclude that on $I_{j}$ we have

$$
\left|\frac{\partial}{\partial t} g\right|=\left|\frac{\partial}{\partial t}\left(g-g_{j}\right)\right| \leq C \sup _{I_{j}}\left|h_{j}\right|_{C^{k}} \leq C \epsilon e^{-\lambda \tau(j-1)}=\frac{C \epsilon}{p^{j-1}} .
$$


Now note that simply integrating over time we see that

$$
\sup _{I_{j}}\left|g-g_{0}\right| \leq 2 \tau \sup _{I_{j} \cup I_{j-1}}\left|\frac{\partial}{\partial t} g\right|+\sup _{I_{j-1}}\left|g-g_{0}\right| .
$$

Applying this estimate inductively we find that

$$
\begin{aligned}
\sup _{I_{j}}\left|g-g_{0}\right| & \leq 2 \tau \sum_{k=1}^{N} \sup _{I_{k} \cup \cdots \cup I_{N}}\left|\frac{\partial}{\partial t} g\right|+\sup _{I_{0}}\left|g-g_{0}\right| \\
& \leq \sum_{k=1}^{\infty} \frac{2 \tau C \epsilon}{p^{k-1}}+\sup _{I_{0}}\left|h_{0}\right| \leq \frac{2 \tau C \epsilon}{p-1}+\sup _{I_{0}}\left|h_{0}\right| .
\end{aligned}
$$

Now we want to choose our constants $\epsilon, \epsilon^{\prime}$ and $\tau$ to derive a contradiction from this inequality. So, choose $\tau$ initially so large that

$$
\frac{1}{c(n) e^{\tau \lambda}}+\frac{2 C \tau}{e^{\tau \lambda}-1}<\frac{1}{C} e^{-\tau \lambda / 4}
$$

where $c(n)$ is a fixed large constant and $C$ is a constant depending only on $g_{0}$.

Now let $\epsilon=\min \left\{\delta(n, \tau), v(n, \tau), \lambda / C_{0}\right\}$ where $\delta(n, \tau)$ is as in Lemma 8.6, $v(n, \tau)$ is as in Lemma 8.8 , and $C_{0}$ is a constant depending only on $g_{0}$ and the dimension which we now make explicit. By Lemma 8.4 we can bound the growth of $L^{2}$ derivatives of $h$, and then by Sobolev embeddings we can bound $C^{k}$ norms. Specifically there exists a constant depending only on $g_{0}$ such that

$$
|h|_{C^{4}}<C e^{C \epsilon \tau}|h(0)|_{C^{0}} .
$$

Then let $C_{0}:=12 C$. Note that if $\epsilon<\lambda / C_{0}$ and we start our flow with some $h\left(t_{0}\right)$ satisfying $\left|h\left(t_{0}\right)\right|_{C^{4}}<(\epsilon / C) e^{-\tau \lambda / 4}$, the solution exists at least on $\left[t_{0}, t_{0}+3 \tau\right)$ and moreover $\sup _{\left[t_{0}, t_{0}+3 \tau\right)}|h(t)|_{C^{4}}<\epsilon$.

Now again using Lemma 8.4 we see that we may choose $\epsilon^{\prime}$ so that the solution exists on $[0,3 \tau]$ and further

$$
\sup _{[0,3 \tau]}|h(t)|_{C^{k}}<\frac{\epsilon}{c(n)} e^{-\tau \lambda} .
$$

Since $\epsilon<\min \{\delta(n, \tau), v(n, \tau)\}$ we can apply 40 to get

$$
\sup _{I_{N}}\left|g-g_{0}\right| \leq \epsilon\left(\frac{1}{c(n) e^{\tau \lambda}}+\frac{2 C \tau}{e^{\tau \lambda}-1}\right) \leq \frac{\epsilon}{C} e^{-\tau \lambda / 4} .
$$

Thus the solution to HCF with initial metric $g(T-\tau)$ exists on an interval of length $3 \tau$ with $|h|_{C^{2}}<\epsilon$, contradicting the maximality of $T$. Thus the solution exists for all time and $\left|g(t)-g_{0}\right|_{C^{k}}<\epsilon$ for all time. Indeed we have decay

$$
\left|g(t)-g_{j}\right| \leq C e^{-\lambda t}
$$

for all $t \in[0, j \tau)$ and for all $j$. Since $\left\{g_{j}\right\}$ is a sequence of Kähler-Einstein metrics with uniform $C^{k}$ bounds, we get a subsequence $g_{j} \rightarrow g_{\infty}$ convergent to a critical metric, with exponential convergence $g(t) \rightarrow g_{\infty}$. 


\section{Further questions}

It bears mentioning again that Theorems 1.1 and 1.2 are both true for solutions to 3 where $Q$ can be any quadratic expression in the torsion. It remains to be discovered which choices of $Q$ lead to flows with favorable existence properties. Ideally one would like to find choices of $Q$ for which either integrability conditions for $\omega$ are preserved, or certain curvature conditions are preserved.

The HCF is similar in some regards to certain renormalization group flows arising in physics where external fields, say Yang-Mills or B-fields, are added to the pure gravity theory and then arise in the flow equations (see for instance [13], [16], [17]). In these flows the torsion is given as an external field, whereas in HCF everything is defined in terms of the metric. A similar case is studied in [3], [10] where a "holonomy flow" is proposed for closed $G_{2}$ structures. Here one evolves the definite three-form $\sigma$ defining the $G_{2}$ structure by the Hodge Laplacian of $\sigma$ taken with the metric induced by $\sigma$. This is a quasilinear equation which bears a certain resemblance to $\mathrm{HCF}$ in that it can be written as "Ricci flow plus torsion", where the torsion is defined in terms of the underlying metric. The techniques of our stability theorem likely apply to show stability of this flow near $G_{2}$-holonomy spaces with negative semidefinite Lichnerowicz operator.

Finally, Hermitian curvature flow may provide a framework for addressing questions on the existence of integrable complex structures. In particular, if one had a complete description of the behavior of this flow for certain geometric conditions and a complete understanding of the limiting objects, one could then describe the manifolds admitting integrable complex structures with Hermitian metrics satisfying the initial geometric conditions. Therefore with strong enough convergence results for this flow one could possibly rule out integrable complex structures on certain manifolds.

\section{Appendix: Variational formulas}

In this appendix we collect variational formulas for quantities related to the curvature and torsion of Hermitian metrics.

Lemma 10.1. Let $g(a)$ be a family of Hermitian metrics compatible with the given complex structure J. Then

$$
\frac{\partial}{\partial a} \Gamma_{i k}^{l}=g^{l \bar{m}} \nabla_{i} h_{k \bar{m}}, \quad \frac{\partial}{\partial a} \Gamma_{\overline{i k}}^{\bar{l}}=g^{\bar{l} m} \nabla_{\bar{i}} h_{m \bar{k}}
$$

Proof. We compute directly in canonical complex coordinates:

$$
\begin{aligned}
\frac{\partial}{\partial a} \Gamma_{i k}^{l} & =\frac{\partial}{\partial a} g^{l \bar{m}}\left(\partial_{i} g_{k \bar{m}}\right)=-h^{l \bar{m}} \partial_{i} g_{k \bar{m}}+g^{l \bar{m}} \partial_{i} h_{k \bar{m}} \\
& =g^{l \bar{m}}\left(\partial_{i} h_{k \bar{m}}-\Gamma_{i k}^{p} h_{p \bar{m}}\right)=g^{l \bar{m}} \nabla_{i} h_{k \bar{m}} .
\end{aligned}
$$

This gives the first formula, and the second follows by conjugation. 
Lemma 10.2. Let $g(a)$ be a family of Hermitian metrics compatible with the given complex structure $J$. Then

$$
\frac{\partial}{\partial a} \Omega_{i \bar{j} k}^{l}=-g^{m \bar{l}} \nabla_{\bar{j}} \nabla_{i} h_{k \bar{m}}, \quad \frac{\partial}{\partial a} \Omega_{i \bar{j} k \bar{l}}=\Omega_{i \bar{j} k}^{m} h_{m \bar{l}}-\nabla_{\bar{j}} \nabla_{i} h_{k \bar{l}} .
$$

Proof. We compute directly:

$$
\begin{aligned}
\frac{\partial}{\partial a} \Omega_{i \overline{j k}}^{l} & =\frac{\partial}{\partial a}\left(-\partial_{\bar{j}} \Gamma_{i k}^{l}\right)=-\partial_{\bar{j}}\left(g^{l \bar{m}} \nabla_{i} h_{k \bar{m}}\right) \\
& =g^{l \bar{p}} \partial_{\bar{j}} g_{\bar{p} q} g^{q \bar{m}} \nabla_{i} h_{k \bar{m}}-g^{l \bar{m}} \partial_{\bar{j}} \nabla_{i} h_{k \bar{m}} \\
& =-g^{m \bar{l}}\left(\partial_{\bar{j}} \nabla_{i} h_{k \bar{m}}-\Gamma_{\bar{j} \bar{m}}^{\bar{p}} \nabla_{i} h_{k \bar{p}}\right)=-g^{m \bar{l}} \nabla_{\bar{j}} \nabla_{i} h_{k \bar{m}}
\end{aligned}
$$

This gives the first formula, and the second follows easily.

Lemma 10.3. Let $g(a)$ be a family of Hermitian metrics compatible with the given complex structure J. Then

$$
\frac{\partial}{\partial a} s=-\Delta \operatorname{tr} h-\left\langle h, S+\operatorname{div}^{\nabla} T-\nabla w\right\rangle .
$$

Proof. We compute using Lemma 10.2

$$
\frac{\partial}{\partial a} s=\frac{\partial}{\partial a} g^{k \bar{l}} S_{k \bar{l}}=-h^{k \bar{l}} S_{k \bar{l}}+g^{k \bar{l}}\left[-\Delta h_{k \bar{l}}-h^{i \bar{j}} \Omega_{i \bar{j} k \bar{l}}+h_{k \bar{m}} S_{\bar{l}}^{\bar{m}}\right]=-\Delta \operatorname{tr} h-\langle h, P\rangle .
$$

The result now follows from Lemma 2.4

Lemma 10.4. Let $g(a)$ be a family of Hermitian metrics compatible with the given complex structure $J$. Then

$$
\begin{aligned}
\frac{\partial}{\partial a} T_{i j \bar{k}} & =\nabla_{i} h_{j \bar{k}}-\nabla_{j} h_{i \bar{k}}+T_{i j}^{m} h_{m \bar{k}}, \\
\frac{\partial}{\partial a} w & =\nabla \operatorname{tr} h-\operatorname{div}^{\nabla} h .
\end{aligned}
$$

Proof. We compute directly:

$$
\begin{aligned}
\frac{\partial}{\partial a} T_{i j \bar{k}} & =\frac{\partial}{\partial a}\left(\partial_{i} g_{j \bar{k}}-\partial_{j} g_{i \bar{k}}\right)=\partial_{i} h_{j \bar{k}}-\partial_{j} h_{i \bar{k}}=\nabla_{i} h_{j \bar{k}}+\Gamma_{i j}^{m} h_{m \bar{k}}-\nabla_{j} h_{i \bar{k}}-\Gamma_{j i}^{p} h_{p \bar{k}} \\
& =\nabla_{i} h_{j \bar{k}}-\nabla_{j} h_{i \bar{k}}+T_{i j}^{m} h_{m \bar{k}} .
\end{aligned}
$$

This gives the first formula. For the second we see that

$$
\begin{aligned}
\frac{\partial}{\partial a} w_{i} & =\frac{\partial}{\partial a} g^{j \bar{k}} T_{i j \bar{k}}=-h^{j \bar{k}} T_{i j \bar{k}}+g^{j \bar{k}}\left(\nabla_{i} h_{j \bar{k}}-\nabla_{j} h_{i \bar{k}}+T_{i j}^{m} h_{m \bar{k}}\right) \\
& =\nabla_{i} \operatorname{tr} h-\operatorname{div}^{\nabla} h_{i} .
\end{aligned}
$$


Lemma 10.5. Let $g(s)$ be a family of Hermitian metrics compatible with the given complex structure $J$. Then

$$
\frac{\partial}{\partial s}|T|^{2}=\left\langle h,-2 Q^{1}+Q^{2}\right\rangle+4\langle\nabla h, T\rangle
$$

where

$$
\langle\nabla h, T\rangle=\frac{1}{2} g^{i \bar{j}} g^{k \bar{l}} g^{m \bar{n}}\left(\nabla_{i} h_{k \bar{n}} T_{\overline{j l} m}+T_{i k \bar{n}} \nabla_{\bar{j}} h_{\bar{l} m}\right) .
$$

Proof. We compute directly:

$$
\begin{aligned}
\frac{\partial}{\partial s} \mid & \left.T\right|^{2}=\frac{\partial}{\partial s} g^{i \bar{p}} g^{j \bar{q}} g^{\bar{k} r} T_{i j \bar{k}} T_{\overline{p q} r} \\
= & -h^{i \bar{p}} g^{j \bar{q}} g^{\bar{k} r} T_{i j \bar{k}} T_{\overline{p q} r}-h^{j \bar{q}} g^{i \bar{p}} g^{\bar{k} r} T_{i j \bar{k}} T_{\overline{p q} r}-h^{\bar{k} r} g^{i \bar{p}} g^{j \bar{q}} T_{i j \bar{k}} T_{\bar{p} \bar{q} r} \\
& \quad+g^{i \bar{p}} g^{j \bar{q}} g^{\bar{k} r}\left[\left(\nabla_{i} h_{j \bar{k}}-\nabla_{j} h_{i \bar{k}}+T_{i j}^{m} h_{m \bar{k}}\right) T_{\overline{p q} r}+T_{i j \bar{k}}\left(\nabla_{\bar{p}} h_{\bar{q} r}-\nabla_{\bar{q}} h_{\bar{p} r}+T_{\overline{p q}}^{\bar{s}} h_{r \bar{s}}\right)\right] \\
= & \left\langle h,-2 Q^{1}+Q^{2}\right\rangle+4\langle\nabla h, T\rangle .
\end{aligned}
$$

Lemma 10.6. Let $g(s)$ be a family of Hermitian metrics compatible with the given complex structure $J$. Then

$$
\frac{\partial}{\partial s}|w|^{2}=-\left\langle h, Q^{3}\right\rangle+2\left\langle\nabla \operatorname{tr} h-\operatorname{div}^{\nabla} h, w\right\rangle .
$$

Proof. In canonical complex coordinates at a point we compute

$$
\begin{aligned}
& \frac{\partial}{\partial s} g^{i \bar{j}} g^{m \bar{n}} g^{r \bar{s}} T_{i m \bar{n}} T_{\bar{j} \bar{s} r} \\
& =-h^{i \bar{j}} g^{m \bar{n}} g^{r \bar{s}} T_{i m \bar{n}} T_{\bar{j} \bar{s} r}-g^{i \bar{j}} h^{m \bar{n}} g^{r \bar{s}} T_{i m \bar{n}} T_{\bar{j} \bar{s} r}-g^{i \bar{j}} g^{m \bar{n}} h^{r \bar{s}} T_{i m \bar{n}} T_{\bar{j} \bar{s} r} \\
& \quad+g^{i \bar{j}} g^{m \bar{n}} g^{r \bar{s}}\left[\left(\nabla_{i} h_{m \bar{n}}-\nabla_{m} h_{i \bar{n}}+T_{i m}^{p} h_{p \bar{n}}\right) T_{\bar{j} \bar{s} r}\right. \\
& \left.\quad+\left(\nabla_{\bar{j}} h_{\bar{s} r}-\nabla_{\bar{s}} h_{\bar{j} r}+T_{\bar{j} \bar{s}}^{\bar{q}} h_{\bar{q} r}\right) T_{i m \bar{n}}\right] \\
& =-\left\langle h, Q^{3}\right\rangle+2\left\langle\nabla \operatorname{tr} h-\operatorname{div}^{\nabla} h, w\right\rangle .
\end{aligned}
$$

Lemma 10.7. Let $g(a)$ be a family of Hermitian metrics compatible with the given complex structure $J$. Then

$$
\frac{\partial}{\partial a} \int_{M} s d V=\int_{M}\left[\left\langle h,-S-\operatorname{div}^{\nabla} T+\nabla w\right\rangle+(\operatorname{tr} h)\left(s-\operatorname{div}^{\nabla} w-|w|^{2}\right)\right] d V .
$$

Proof. From Lemma 10.3 we obtain

$$
\frac{\partial}{\partial a} \int_{M} s d V=\int_{M}\left[-\Delta \operatorname{tr} h+\left\langle h,-S-\operatorname{div}^{\nabla} T+\nabla w\right\rangle+s \operatorname{tr} h\right] d V .
$$

Now by Lemma 10.11 we see

$$
\int_{M}-\Delta \operatorname{tr} h d V=\int_{M}(\operatorname{tr} h)\left(-\operatorname{div}^{\nabla} w-|w|^{2}\right) d V,
$$

and the result follows. 
Lemma 10.8. Let $g(a)$ be a family of Hermitian metrics compatible with the given complex structure $J$. Then

$$
\frac{\partial}{\partial a} \int_{M}|T|^{2} d V=\int_{M}\left[\left\langle h,-2 Q^{1}+Q^{2}-4 Q^{4}-4 \operatorname{div}^{\nabla} T\right\rangle+(\operatorname{tr} h)|T|^{2}\right] d V .
$$

Proof. We directly compute using Lemma 10.5

$$
\frac{\partial}{\partial a} \int_{M}|T|^{2} d V=\int_{M}\left[\left\langle h,-2 Q^{1}+Q^{2}\right\rangle+4\langle\nabla h, T\rangle+(\operatorname{tr} h)|T|^{2}\right] d V .
$$

We now integrate by parts:

$$
\begin{aligned}
\int_{M}\langle\nabla h, T\rangle d V & =\int_{M} g^{i \bar{p}} g^{j \bar{q}} g^{\bar{k} r} \nabla_{i} h_{j \bar{k}} T_{\overline{p q} r} d V \\
& =\int_{M} g^{i \bar{p}} g^{j \bar{q}} g^{\bar{k} r}\left(\partial_{i} h_{j \bar{k}}-\Gamma_{i j}^{S} h_{s \bar{k}}\right) T_{\bar{p} \bar{q}} d V \\
& =-\int_{M} h_{j \bar{k}} \partial_{i}\left(g^{i \bar{p}} g^{j \bar{q}} g^{\bar{k} r} T_{\overline{p q} r} d V\right)+g^{i \bar{p}} g^{j \bar{q}} g^{\bar{k} r} \Gamma_{i j}^{s} h_{s \bar{k}} T_{\overline{p q} r} d V \\
& =\int_{M} h_{j \bar{k}}\left[g^{i \bar{n}} \partial_{i} g_{m \bar{n}} g^{m \bar{p}} g^{j \bar{q}} g^{\bar{k} r} T_{\overline{p q} r}+g^{i \bar{p}} g^{j \bar{n}} \partial_{i} g_{m \bar{n}} g^{m \bar{q}} g^{\bar{k} r} T_{\overline{p q} r}\right. \\
& \quad+g^{i \bar{p}} g^{j \bar{q}} g^{\bar{k} m} \partial_{i} g_{m \bar{n}} g^{\bar{n} r} T_{\overline{p q} r}-g^{i \bar{p}} g^{j \bar{q}} g^{\bar{k} r} \partial_{i} T_{\overline{p q} r} \\
& \quad-g^{i \bar{p}} g^{j \bar{q}} g^{\bar{k} r} T_{\overline{p q} r} g^{m \bar{n}} \partial_{i} g_{m \bar{n}} \\
& \left.-g^{m \bar{p}} g^{n \bar{q}} g^{\bar{k} r} \Gamma_{m n}^{j} T_{\overline{p q} r}\right] d V \\
= & \int_{M}\left\langle h,-\operatorname{div}^{\nabla} T-Q^{4}\right\rangle d V .
\end{aligned}
$$

Plugging in this simplification yields the result.

Lemma 10.9. Let $g(a)$ be a family of Hermitian metrics compatible with the given complex structure $J$. Then

$$
\frac{\partial}{\partial a} \int_{M}|w|^{2} d V=\int_{M}\left[\left\langle h, Q^{3}+2 \nabla w\right\rangle+(\operatorname{tr} h)\left[-2 \operatorname{div}^{\nabla} w-|w|^{2}\right]\right] d V .
$$

Proof. We directly compute using Lemma 10.6 and integrating by parts using Lemmas 10.10 and 10.12 .

$$
\begin{aligned}
\frac{\partial}{\partial a} \int_{M}|w|^{2} d V & =\int_{M}\left[\left\langle h,-Q^{3}\right\rangle+2\left\langle\nabla \operatorname{tr} h-\operatorname{div}^{\nabla} h, w\right\rangle+(\operatorname{tr} h)|w|^{2}\right] d V \\
= & \int_{M}\left[\left\langle h,-Q^{3}\right\rangle+2(\operatorname{tr} h)\left[-\operatorname{div}^{\nabla} w-|w|^{2}\right]\right. \\
& \left.+2\left\langle h, \nabla w+Q^{3}\right\rangle+(\operatorname{tr} h)|w|^{2}\right] d V .
\end{aligned}
$$


Lemma 10.10. Given $\phi \in C^{\infty}(M)$ and $\alpha \in T^{1,0}(M)$ we have

$$
\int_{M}\langle\nabla \phi, \alpha\rangle=\int_{M} \phi\left[-\operatorname{div}^{\nabla} \alpha-\langle w, \alpha\rangle\right] d V .
$$

Proof. We directly compute

$$
\begin{aligned}
\int_{M}\langle\nabla \phi, \alpha\rangle d V & =\int_{M} g^{i \bar{j}} \partial_{i} \phi \alpha_{\bar{j}} d V=-\int_{M} \phi \partial_{i}\left[g^{i \bar{j}} \alpha_{\bar{j}} d V\right] \\
& =\int_{M} \phi\left[g^{i \bar{s}} \partial_{i} g_{r \bar{s}} g^{r \bar{j}} \alpha_{\bar{j}}-g^{i \bar{j}} \partial_{i} \alpha_{\bar{j}}-g^{i \bar{j}} \alpha_{\bar{j}} g^{p \bar{q}} \partial_{i} g_{p \bar{q}}\right] d V \\
& =\int_{M} \phi\left[-\operatorname{div}^{\nabla} \alpha-\langle w, \alpha\rangle\right] d V .
\end{aligned}
$$

Lemma 10.11. Given $\phi \in C^{\infty}(M)$ we have

$$
\int_{M} \Delta \phi d V=\int_{M} \phi\left[\operatorname{div}^{\nabla} w+|w|^{2}\right] d V .
$$

Proof. We directly compute

$$
\begin{aligned}
\int_{M} \Delta \phi d V & =\int_{M} g^{i \bar{j}} \bar{\partial}_{\bar{j}} \partial_{i} \phi d V=-\int_{M} \partial_{i} \phi \partial_{\bar{j}}\left(g^{i \bar{j}} d V\right) \\
& =\int_{M} \partial_{i} \phi\left[g^{i \bar{s}} \partial_{\bar{j}} g_{r \bar{s}} g^{r \bar{j}}-g^{i \bar{j}} g^{p \bar{q}} \partial_{\bar{j}} g_{p \bar{q}}\right] d V=\int_{M} \partial_{i} \phi\left[g^{i \bar{j}} g^{p \bar{q}} T_{\bar{q} \bar{j} p}\right] d V \\
& =-\int_{M}\langle\nabla \phi, w\rangle d V=\int_{M} \phi\left[\operatorname{div}^{\nabla} w+|w|^{2}\right] d V .
\end{aligned}
$$

For the last equality we applied Lemma 10.10

Lemma 10.12. Given $\beta \in T^{1,0}(M)$ and $h \in \operatorname{Sym}^{1,1}(M)$ we have

$$
\int_{M}\left\langle\operatorname{div}^{\nabla} h, \beta\right\rangle d V=\int_{M}[\langle h,-\nabla \beta-w \otimes \beta\rangle] d V .
$$

Proof.

$$
\begin{aligned}
& \int_{M}\left\langle\operatorname{div}^{\nabla} h, \beta\right\rangle d V=\int_{M} g^{\bar{j} l} g^{\bar{k} i} \nabla_{\bar{k}} h_{i \bar{j}} \beta_{l} d V=\int_{M} g^{i \bar{k}} g^{l \bar{j}}\left[\partial_{\bar{k}} h_{i \bar{j}}-\Gamma_{\overline{k j}}^{\bar{p}} h_{i \bar{p}}\right] \beta_{l} d V \\
& =-\int_{M} h_{i \bar{j}} \partial_{\bar{k}}\left[g^{i \bar{k}} g^{l \bar{j}} \beta_{l} d V\right]+\int_{M} g^{i \bar{k}} g^{l \bar{j}} \Gamma_{\overline{k j}}^{\bar{p}} h_{i \bar{p}} \beta_{l} d V \\
& =\int_{M} h_{i \bar{j}}\left[g^{i \bar{s}} \partial_{\bar{k}} g_{r \bar{s}} g^{r \bar{k}} g^{l \bar{j}} \beta_{l}+g^{i \bar{k}} g^{l \bar{s}} \partial_{\bar{k}} g_{r \bar{s}} g^{r \bar{j}} \beta_{l}-g^{i \bar{k}} g^{l \bar{j}} \partial_{\bar{k}} \beta_{l}-g^{i \bar{k}} g^{l \bar{j}} g^{r \bar{s}} \partial_{\bar{k}} g_{r \bar{s}} \beta_{l}\right] d V \\
& \quad-\int_{M} g^{i \bar{k}} g^{l \bar{j}} \Gamma_{\bar{k} \bar{p}}^{\bar{p}} h_{i \bar{p}} \beta_{l} d V \\
& =\int_{M}[\langle h,-\nabla \beta-w \otimes \beta\rangle] d V .
\end{aligned}
$$


Acknowledgments. The first author would like to thank Aaron Naber, Yanir Rubinstein, and Jeff Viaclovsky for interesting conversations.

The first author was supported by the National Science Foundation via DMS-0703660. The second author was partly supported by the National Science Foundation via DMS-0703985 and DMS-0804095.

\section{References}

[1] Aubin, T.: Some Nonlinear Problems in Riemannian Geometry. Springer, Berlin (1998) Zbl 0896.53003 MR 1636569

[2] Besse, A.: Einstein Manifolds. Springer, Berlin (1987) Zbl 0613.53001 MR 2371700

[3] Bryant, R.: Some remarks on $G_{2}$-structures. In: Proceedings of Gökova Geometry-Topology Conference 2005, 75-109 (2006) Zbl 1115.53018 MR 2282011

[4] Catenacci, R., Marzuoli, A.: A note on a hermitian analog of Einstein spaces. Ann. Inst. H. Poincaré 40, 151-157 (1984) Zbl 0538.53051 MR 0747199

[5] Cheeger, J., Tian, G.: On the cone structure at infinity of Ricci flat manifolds with Euclidean volume growth and quadratic curvature decay. Invent. Math. 118, 493-571 (1994) Zbl 0814.53034 MR 1296356

[6] Chow, B., Lu, P., Ni, L.: Hamilton's Ricci Flow. Amer. Math. Soc. and Science Press (2006) Zbl 1118.53001 MR 2274812

[7] DeTurck, D., Kazdan, L.: Some regularity theorems in Riemannian geometry. Ann. Sci. École Norm. Sup. (4) 14, 249-260 (1981) Zbl 0486.53014 MR 0644518

[8] Gauduchon, P.: La 1-forme de torsion d'une variété hermitienne compacte. Math. Ann. 267, 495-518 (1984) Zbl 0523.53059 MR 0742896

[9] Gauduchon, P.: Variation des courbures scalaires en géométrie hermitienne. C. R. Acad. Sci. Paris Sér. A-B 290, 327-330 (1980) Zbl 0422.58015 MR 0567760

[10] Hitchin, N.: The geometry of three-forms in 6 and 7 dimensions. J. Differential Geom. 55, 547-576 (2000) Zbl 1036.53042 MR 1863733

[11] Kobayashi, S. Nomizu, K.: Foundations of Differential Geometry. Vol. 1, Wiley-Interscience, London (1963) Zbl 0119.37502 MR 1393940

[12] Morrow, J., Kodaira, K.: Complex Manifolds. AMS Chelsea Publ. (2006) Zbl 1087.32501 MR 2214741

[13] Oliynyk, T., Suneeta, V., Woolgar, E.: A gradient flow for worldsheet nonlinear sigma models. Nucl. Phys. B 739, 441-458 (2006) Zbl 1109.81058 MR 2214659

[14] Sesum, N.: Linear and dynamic stability of Ricci flat metrics. Duke Math. J. 133, 1-26 (2006) Zbl 1103.53040 MR 2219268

[15] Simon, L.: Asymptotics for a class of non-linear evolution equations, with applications to geometric problems. Ann. of Math. 118, 525-571 (1983) Zbl 0549.35071 MR 0727703

[16] Streets, J.: Regularity and expanding entropy for connection Ricci flow. J. Geom. Phys. 58, 900-912 (2008) Zbl 1144.53326 MR 2426247

[17] Streets, J.: Singularities of renormalization group flows. J. Geom. Phys. 59, 8-16 (2009) Zbl 1153.53329 MR 2479257

[18] Tian, G.: Canonical Metrics in Kähler Geometry. Birkhäuser (2000) Zbl 0978.53002 MR 1787650

[19] Vaisman, I.: On some variational problems for 2-dimensional Hermitian metrics. Ann. Global Anal. Geom. 8, 137-145 (1990) Zbl 0721.53062 MR 1088507 